\title{
Online denoising of eye-blinks in electroencephalography
}

Quentin Barthélemy ${ }^{1, *}$, Louis Mayaud ${ }^{1}$, Yann Renard ${ }^{1}$, Daekeun Kim², Seung-Wan Kang ${ }^{2}$, Jay Gunkelman ${ }^{3}$, Marco Congedo ${ }^{4}$

${ }^{1}$ Mensia Technologies, 75015 Paris, France

${ }^{2}$ Seoul National University, Seoul, Korea

${ }^{3}$ Brain Science International, Pleasanton, USA

${ }^{4}$ GIPSA-Lab, CNRS, Grenoble Alpes University, Grenoble Institute of Technology, 38402 Saint Martin d'Hères, France

* Corresponding Author: Mensia Technologies, 130 rue de Lourmel, 75015 Paris, France, qb@mensiatech.com (Q. Barthélemy)

Running title: Online denoising of eye-blinks in EEG 


\section{Online denoising of eye-blinks in electroencephalography}

\section{Abstract \\ Objective}

Due to its high temporal resolution, electroencephalography (EEG) has become a broadly-used technology for real-time brain monitoring applications such as neurofeedback (NFB) and brain-computer interfaces (BCI). However, since EEG signals are prone to artifacts, denoising is a crucial step that enables adequate subsequent data processing and interpretation. The aim of this study is to compare manual denoising to unsupervised online denoising, which is essential to real-time applications.

\section{Methods}

Denoising EEG for real-time applications requires the implementation of unsupervised and online methods. In order to permit genericity, these methods should not rely on electrooculography (EOG) traces nor on temporal/spatial templates of the artifacts. Two blind source separation (BSS) methods are analyzed in this paper with the aim of automatically correcting online eye blink artifacts: the algorithm for multiple unknown signals extraction (AMUSE) and the approximate joint diagonalization of Fourier cospectra (AJDC). The chosen gold standard is a manual review of the EEG database carried out retrospectively by a human operator. Comparison is carried out using the spectral properties of the continuous EEG and event related potentials (ERP).

\section{Results and conclusion}

The AJDC algorithm addresses limitations observed in AMUSE and outperforms it. No statistical difference is found between the manual and automatic approaches on a database composed of 15 healthy individuals, paving the way for an automated, operator-independent, and real-time eye blink correction technique.

Keywords: blind source separation, denoising, electroencephalography, eye blink, online, unsupervised 


\section{Débruitage en ligne de clignements des yeux en électro-encéphalographie}

\section{Résumé}

\section{Objectifs}

Grâce à sa haute résolution temporelle, l'électro-encéphalographie (EEG) est devenue une technologie répandue pour des applications de suivi de l'activité cérébrale en temps-réel, comme le neurofeedback (NFB) et les interfaces cerveau-machine (ICM). Cependant, les signaux EEG étant sensibles aux artéfacts, le débruitage est une étape essentielle qui permet un traitement et une interprétation convenables de la donnée. Le but de cette étude est de comparer les méthodes de débruitage manuel et de débruitage en ligne non-supervisé, essentiel pour les applications temps-réel.

\section{Méthodes}

Débruiter l'EEG pour des applications temps-réel exige l'implémentation de méthodes de débruitage en ligne non-supervisées. De plus, afin d'être génériques, ces méthodes ne devraient pas s'appuyer sur une référence électro-oculographique (EOG), ni sur un modèle temporel ou spatial des artéfacts. Deux méthodes de séparation aveugle de source (SAS) sont analysées dans ce papier avec le but d'automatiquement corriger en ligne les artéfacts de clignements des yeux: l'algorithme d'extraction de multiples signaux inconnus (AEMSI) et la diagonalisation conjointe approchée des cospectres de Fourier (DCAC). Le test de référence choisi est l'examen manuel de la base de données complète, réalisée rétrospectivement par un opérateur humain. La comparaison est réalisée en utilisant les propriétés spectrales de l'EEG continu et les potentiels évoqués.

\section{Résultats et conclusion}

L'algorithme DCAC résout les limitations observes dans AEMSI et s'avère plus performant. Aucune différence statistique n'est trouvée entre les approches manuelle et automatique sur une base de données composée de 15 sujets sains, ouvrant la voie pour une technique de correction de clignement des yeux automatique, sans opérateur et temps-réel.

Mots clés: clignement des yeux, débruitage, électroencéphalographie, en ligne, non-supervisé, séparation aveugle de sources. 


\section{Introduction}

Electroencephalography (EEG) is a non-invasive measure of cerebral electrical potentials recorded at several scalp locations. Due to its high temporal resolution and portability, EEG has become a broadly-used technology for brain monitoring [45]. Unfortunately, EEG time-series are prone to several different types of artifacts, i.e., electric potentials that are not generated by the brain. Artifacts may have biological, instrumental or environmental origin. Occasionally, their energy may be some order of magnitude greater than that of the neurophysiological signal of interest [12] [76]. In this context, proper denoising is a crucial step for EEG data analysis [30]. Denoising refers to the removal of components/sources that are not considered "useful" or "informative" with respect to the application. Many methods have been developed for this purpose [20] [73] [82] [29] [81]. With respect to their data processing procedure, the denoising methods can be classified into four categories, which we have termed: manual, offline, block-online and online (Fig. 1). These are defined below.

- Manual: The complete recording is available, from beginning to end. A human operator inspects the recording on an epoch-by-epoch basis and marks artifactual epochs. Epochs containing artifacts are either entirely rejected (approach not considered in this study), or the artifactual sources are rejected, thus providing denoised/corrected epochs [84] [35] [80].

- Offline: The complete recording is available, from beginning to end, and is processed automatically by an expert algorithm, such as FASTER [57], MARA [90], ADJUST [51], PureEEG [27], SASICA [8] or Autoreject [32].

- Block-online: The recording is processed automatically on an epoch-by-epoch basis, with epoch length greater than $500 \mathrm{~ms}$, for instance, as in LAMIC [56] or FORCe [16]. Block-online unsupervised approaches typically require blocks (sometimes called trials) of about $1 \mathrm{~s}$ to $4 \mathrm{~s}$ length [56] [23] [16].

- Online: The recording is processed online as before, but instantaneously. Although these methods may work on a sample-by-sample basis, the EEG acquisition in practice is carried out by buffered blocks. These methods are different from those belonging to the previous category in that the block size may be very small (typically, 10 to $50 \mathrm{~ms}$ ). In this way, the processing may be considered as "real-time".

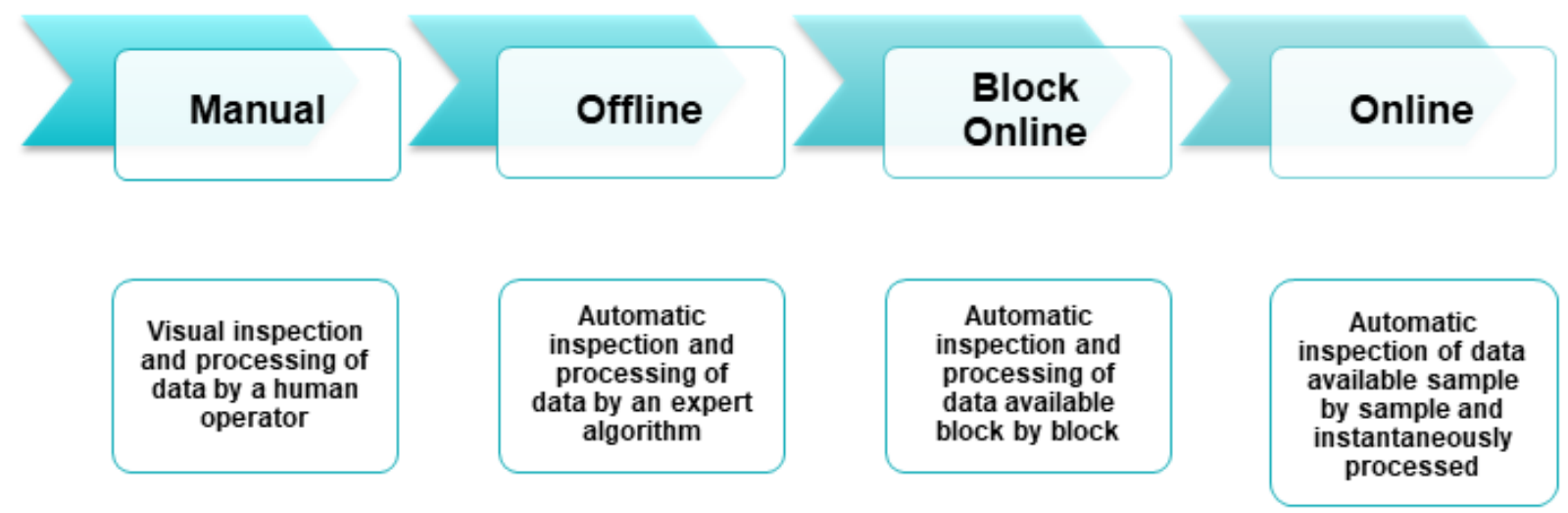

Fig. 1 Current available methods for denoising EEG signals displayed in increasing order of technical difficulty: manual, offline, block-online and online.

The manual procedure is often considered the gold standard in terms of quality [35]. However, it is fully supervised and thus time-consuming. It also introduces an operator-dependent bias with consequent problems of consistency and repeatability. Automated offline approaches can exploit the whole EEG database, and thus are 
generally efficient in addition to being consistent [57], [8]. Some of these offline approaches require an initial supervised calibration to learn detection thresholds [51] or classifiers [90] [39] [66]. Obviously, neither the manual nor the offline procedure is of any use in real-time applications such as neurofeedback (NFB) [1] and brain-computer interfaces (BCI) [45]. To be useful in these real-time applications, the denoising procedure must minimize the feedback delay in order to maximize processing speed, in addition to being unsupervised. The ability of an automated denoising procedure to operate on a sample-by-sample basis is thus instrumental for these applications. The task is challenging and the difficulty is exacerbated by the high variability and nonstationarity of artifacts.

In this article, we are interested in fully unsupervised denoising methods that can operate on a sample-by-sample basis. We focus on the denoising of eye blinks because these artifacts are ubiquitous and inevitable in eyes-open EEG recordings. In contrast to eye movements, eye-blinks generate potentials through the eyelid sliding down over the positively charged cornea [43] [44]. They produce shifts of the electric fields that propagate across the whole head and their amplitude can be one order of magnitude larger than the activity generated by the brain [24]. Eye-blinks may affect the signal amplitude over the entire scalp [30] with a strong energy (decreasing in frequency with a power law decay) and a typical frontal-to-posterior decaying gradient (Fig. 2). The blink waveform shows a large inter-subject variability. Furthermore, voluntary blinks are larger in amplitude and longer in duration than natural ones [43].

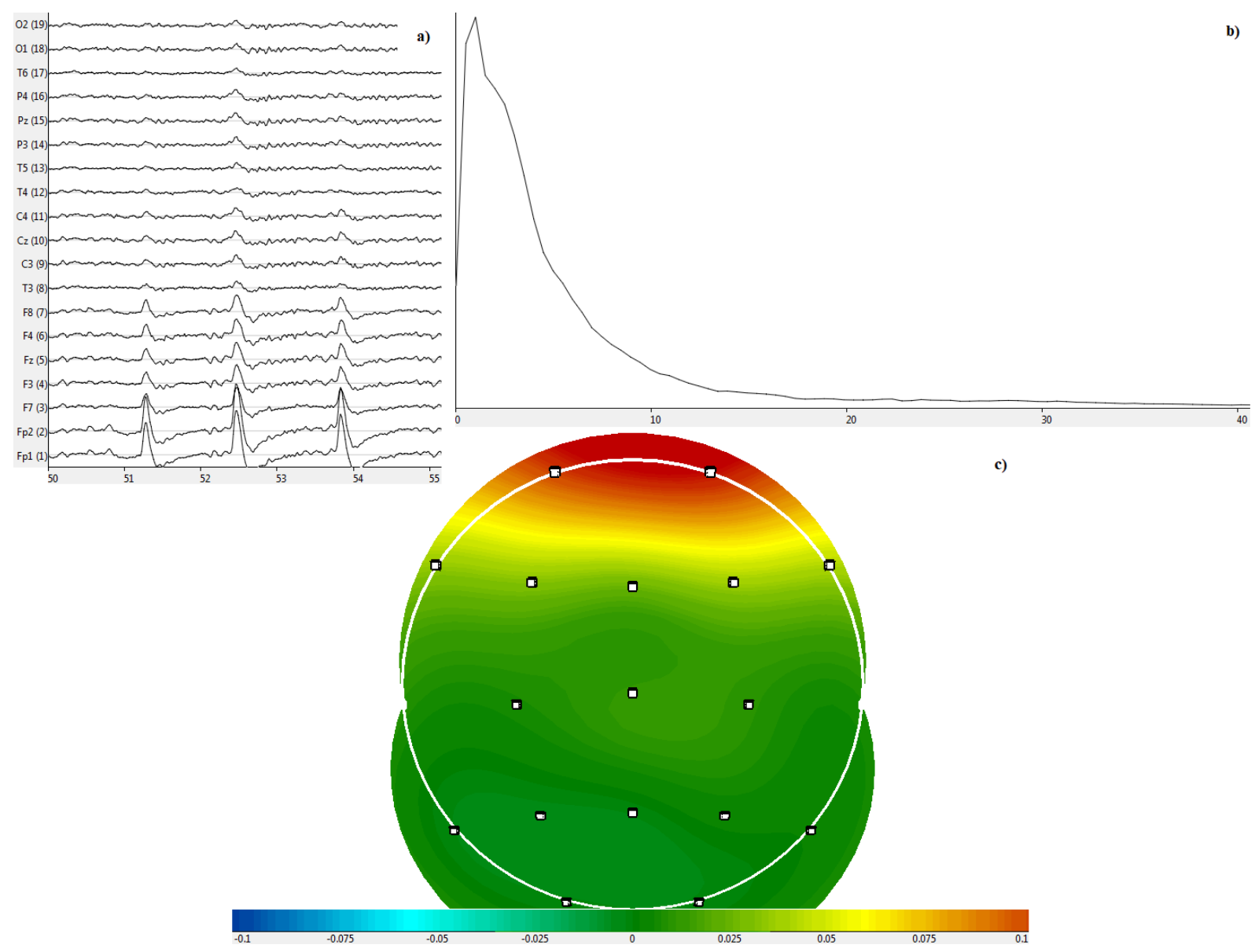

Fig. 2 a) Example of EEG recording with eye blinks; very strong eye blinks in frontal channels, with time in seconds on the $\mathrm{x}$-axis and channels name on the y-axis. b) The spectral density of the blink source identified with the AJDC technique (see next section), with frequencies in $\mathrm{Hz}$ on the x-axis. c) The associated scalp topography of the blink source, indicating electrode location with white squares. 
Denoising eye-blinks is crucial for NFB and BCI applications. In NFB protocols, EEG is analyzed in real-time and transformed into a metric that is instantaneously fed back to the subject. This metric is based on sets of target frequency band power, with the goal for subjects to learn and ultimately regulate the amplitudes or ratios of such powers. While eye-blinks have a strong energy in delta and theta bands, the blinks will still be present in alpha and beta bands [22] [26] [46] [47]. Consequently, the energy of uncorrected blinks can overlap the target frequency bands of NFB, and modify the feedback stream [53]. In BCI, artifacts must not be utilized mistakenly (i) during the training step, to be sure that neurophysiological activity controls the BCI instead of artifacts, and

(ii) during the online step, to avoid artifacts interfering with the brain control. Artifacts kept in these two steps degrade the performance of the BCI [49] [89] [16] [67] [37].

A powerful method for denoising eye blinks is blind source separation (BSS). The aim of BSS is to estimate unknown sources from observed channels, which are the result of an unknown mixing process [9] [11]. The method is called blind because neither the sources nor the mixing process are assumed to be known. BSS denoising consists of decomposing the signal into several source components, rejecting sources identified as artifacts, then reconstructing the signal.

The purpose of this study is to compare the performance of two blind source separation (BSS) methods: the algorithm for multiple unknown signals extraction (AMUSE) [79] and the approximate joint diagonalization of Fourier cospectra (AJDC) [12]. Both methods are unsupervised and are suitable for eye blink denoising on a sample-by-sample basis. As a benchmark method, we chose a manual denoising procedure. We analyze a database of resting-state continuous recordings and event-related recordings of 15 subjects. To compare manual and automatic denoising, spectral and spatial correlations are estimated between manual and automatic denoised power spectra, and event related potentials (ERP) enhancement is estimated in terms of signal-to-noise ratio (SNR).

\section{Materials and methods}

\section{Participants and EEG recordings}

EEG data of 15 healthy subjects aged between 7 and 80 years were used in this study. The data is part of a normative database previously acquired as a standard reference by the Data Center for Korean EEG, Seoul National University, Korea. The exclusion criteria for the database were: history of pathology during the perinatal period, history of neurological or mental diseases, head injuries or convulsive/paroxysmal activity, current serious behavioral problems or current consumption of CNS-active medications.

EEG was recorded from the subjects during a visual continuous performance task (VCPT) designed for ERP studies [63], in addition to an eyes closed (EC) and eyes open (EO) resting condition. The EEG was recorded by a Mitsar-EEG 202 digital electroencephalograph (Mitsar Ltd., St. Petersburg, Russia) with an electro-cap (Electro-cap International Inc., Eaton, Ohio, USA) equipped with 19 electrodes placed according to the international 10-20 system [33]: Fp1, Fp2, F7, F3, Fz, F4, F8, T3, C3, Cz, C4, T4, T5, P3, Pz, P4, T6, O1, and O2. The reference electrodes were placed on the mastoids and the ground electrode was placed at the Fpz derivation. Electrode impedance at all electrodes did not exceed $5 \mathrm{k} \Omega$. The EEG sampling rate was $250 \mathrm{~Hz}$ and the amplifier band-pass was set to $0.53-50 \mathrm{~Hz}$. The participants were asked to sit up straight and watch a 17-inch computer monitor placed $1.5 \mathrm{~m}$ in front of them. For the eyes open condition, participants were asked to sit in the 
chair comfortably and to move as little as possible. For the eyes closed condition, participants were asked to avoid falling asleep or being drowsy. The length of the recording for the VCPT task was 21 minutes. For both the EO and EC conditions, the length was 4 minutes.

\section{Ethical statements}

All procedures performed in studies involving human participants were in accordance with the ethical standards of the institutional and/or national research committee and with the 1964 Helsinki declaration and its later amendments or comparable ethical standards. Informed consent was obtained from all individual participants included in the study. The data used in this paper was collected after approval from the IRB (Institutional Review Board) committee in Seoul National University, Korea.

\section{Procedures}

In order to compare and contrast the performance of different denoising techniques, we decided to quantify the SNR of two known physiological components: the alpha rhythm elicited in occipital areas during EO condition and the EPR following the presentation of a visual or auditory stimulation. Our hypothesis is that the amplitude of the SNR extracted from signals pre-processed with different techniques should reflect the effectiveness of those techniques. More precisely, a more accurate rejection of eye blinks should translate into a higher EO or ERP SNR. In the literature, the ERP task is a common way to compare denoising procedures [24] [13] [56] [50] [57] [82] since grand average ERPs are damaged by ocular artifacts.

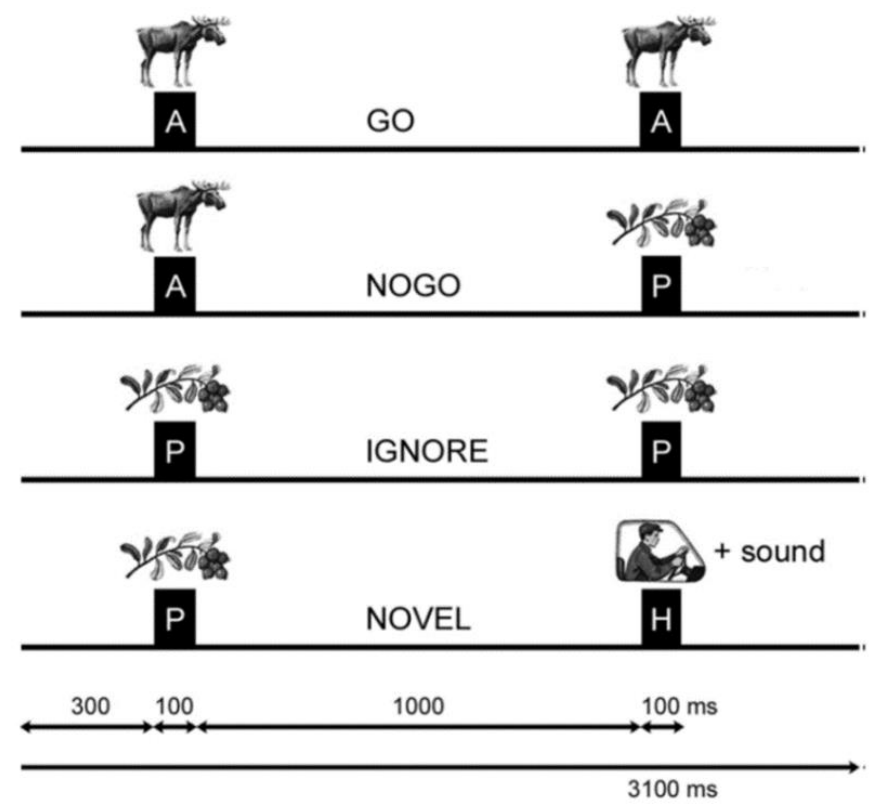

Fig. 3 Description of the four pairs of stimuli forming the Go-NoGo protocol, with stimulus duration and inter-stimulus intervals in ms: conditions Go, NoGo, Ignore and Novel.

The ERP task is a Go-NoGo paradigm using four pairs of stimuli presented in succession with a 1s inter-stimulus interval (Fig. 3). The four possible pairs are:

- Go condition: animal (A) followed by animal. Participants are required to press a button as quickly as possible. 
- NoGo condition: animal followed by plant (P). Participants are required not to press the button.

- Ignore condition: plant followed by plant. Participants are required not to press the button.

- Novel condition: plant followed by human $(\mathrm{H})$, plus a novelty sound. Participants are required not to press the button.

Each condition was presented 100 times, allowing for the recording of 400 ERP trials in total. After a careful visual inspection of the recordings, data from subject 3 was excluded from the ensuing analysis because of the bad quality of the manually denoised recording. Consequently, data of 14 participants were included in the ensuing analysis.

\section{Description of denoising techniques}

\section{Blind source separation}

In BSS we assume that the observed recordings at the scalp are the result of an unknown mixture of unknown sources [9] [11]. Because of a number of physiological and physical reasons listed in [12], the multichannel EEG signal recorded at $\mathrm{N}$ channels/sensors $\mathrm{X} \in \mathbb{R}^{\mathrm{N}}$ can be modeled as a linear (instantaneous) combination of $\mathrm{M} \leq \mathrm{N}$ independent sources $S \in \mathbb{R}^{\mathrm{M}}$, such as

$\mathrm{X}=\mathrm{AS}$,

where $A \in \mathbb{R}^{\mathrm{N} \times \mathrm{M}}$ is the mixing matrix, considered constant throughout the recording. The goal of BSS is to estimate the separating/demixing matrix $\mathrm{B} \in \mathbb{R}^{\mathrm{M} \times \mathrm{N}}$ allowing source estimation

$\mathrm{S}=\mathrm{B} \mathrm{X}$

with B being the estimated pseudo-inverse of (unknown) matrix A. According to BSS theory, the source process can be estimated up to a permutation and scaling factor (amplitude and sign). Many BSS methods exist to estimate the separating matrix imposing statistical properties to sources, including independent component analysis (ICA) [10], which is based on the estimation of higher order statistics (HOS) [7], and methods based on second order statistics (SOS) [4]. The cleaned signal is obtained by rejecting sources identified as artifacts by the diagonal activation matrix $\mathrm{D} \in \mathbb{R}^{\mathrm{M} \times \mathrm{M}}$ whose diagonal elements are 0 for artifact components and 1 otherwise, such as

$\widetilde{\mathrm{X}}=\mathrm{A}$ D S .

The process of BSS denoising is summarized schematically in Fig. 4. In BSS, the estimation of the separating/demixing matrix $\mathrm{B}$ is unsupervised, but this is not the case for the activation matrix $\mathrm{D}$, which is needed to identify the artifactual sources; this can be obtained either manually in a supervised fashion (cf. manual denoising in Section 0) or automatically with additionally processing.

For eye blink denoising, in [25], [14], [54], [34], [86], [21], [69], [38], [68] and [61], several automatic methods have been proposed to identify the eye blink component. All these methods require the recording of 
electrooculographic (EOG) traces in addition to EEG using one or more additional electrodes next to the eyeball. The availability of EOG channels makes the detection easier; however this is not always possible since many EEG headsets do not include EOG channels. In this paper we are interested in methods based on EEG channels only. Among those, some methods use artifact templates/references extracted on a database: temporal templates [34] [56] or spatial templates [42] [85] [5]. Temporal templates are sensitive to sampling rate and filtering characteristics (filter order, cut-off frequencies), whereas spatial templates are specific to a particular EEG spatial configuration, i.e., the configuration of the channels of the headset used in the database. Other methods include prior knowledge in a semi-blind source separation (SBSS), using temporal constraints [31] or spatial constraints [28] [55]. Spatial constraints can be computed from a Parallel Factor analysis [87] applied on a space-time-frequency decomposition of eye-blink contaminated EEG recordings [55]. These constraints, however, have the same limitations as templates. Alternatively, eye-blink component identification can be performed by machine learning [71] [40] [6] [18] [70] [90] [83] [66]: spectral, temporal and spatial features are extracted on a database and then a classifier decides if a component is to be considered as an artifact or not. As a matter of fact, all these methods require a supervised training step applied on extensive labelled recordings. This is another limitation we wish to avoid.

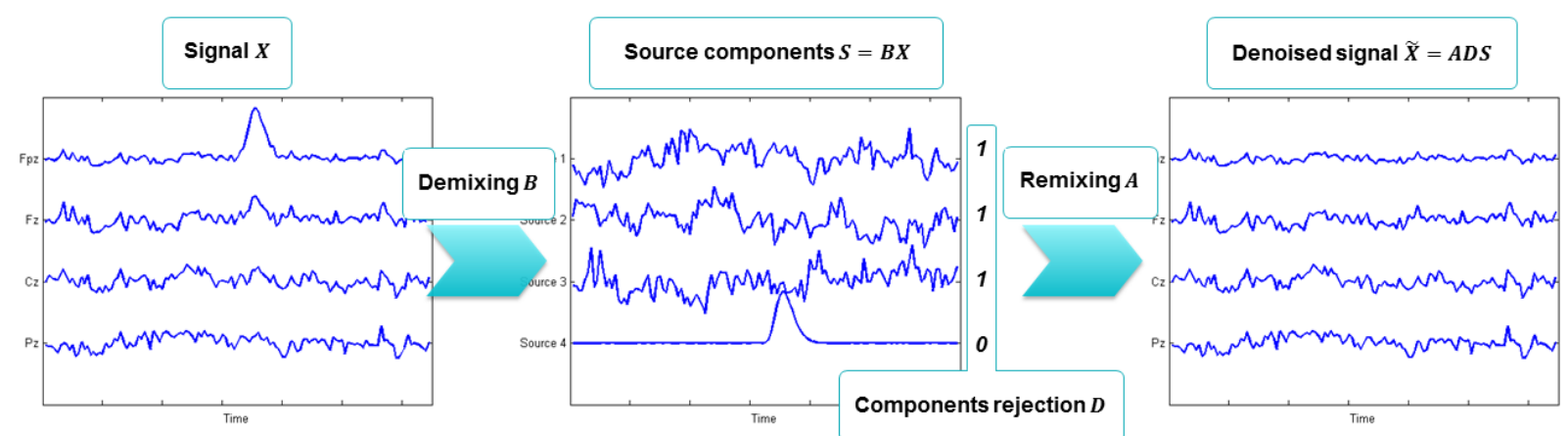

Fig. 4 Illustration of the BSS denoising concept: first, the signal $X$ is decomposed in sources components using the estimated demixing matrix $B$; then, artifactual sources are rejected using activation matrix $D$; and finally, the signal is projected back into the sensor space using the estimation of the mixing matrix $A$ (the pseudo-inverse of $\mathrm{B}$ ), providing the sought denoised signal $\tilde{X}$.

In this article, we are interested in an unsupervised online eye-blink denoising procedure that does not make use of EOG traces or an extensive training database. Moreover, we require the procedure be reference-free, i.e., without a spatial or temporal template that is dependent on the headset, and potentially specific to the training subject(s) [43]. Such a generic method would thus be compatible with any headset. In order to achieve this, we allow only a non-supervised self-calibration step, i.e., a small segment of signal can be used to learn relevant features. Consequently, this type of denoising can be applied on a single signal/trial, as well as on a complete database.

\section{Manual denoising}

Manual denoising was carried out using the WinEEG software (Nova Tech EEG, Mesa, United States). The application filters EEG and removes artifacts using a supervised ICA technique [35] known as Infomax [3] [41]. To remove artifacts, the operator scrolls the data and selects the artifactual epochs. ICA is performed on artifactual epochs, and a visual inspection of source waveforms and topographies is then made by the operator to 
select artifactual sources, which are rejected specifying the diagonal activation matrix D described above. The remaining sources are then projected back into the sensors" space in order to recover the "de-artifacted" epoch. This denoising approach is called "manual" because artifactual epochs and artifactual sources are identified by an operator.

\section{Online unsupervised denoising}

Online BSS denoising was carried out with the NeuroRT Studio software (Mensia Technologies, Paris, France), a complemented and certified version of the OpenViBE software [62], which was initially designed for real-time analysis of EEG signals, BCI and virtual reality (http://openvibe.inria.fr/). In NeuroRT Studio, two denoising plugins are dedicated to the online removal of eye blink artifacts: the first one based on maximum noise fraction (MNF) [72], which is equivalent to the well-known AMUSE [79], and the other based on AJDC [12]. For research reproducibility, these two plugins are available in NeuroRT Studio. The two denoising methods can operate on a sample-by-sample basis; denoising is thus independent from the block/epoch/window duration of the input signal. Of course, such methods may be used to denoise the EEG stream instantaneously (online), with a short delay (block-online) or retrospectively (offline).

AMUSE and AJDC make the same hypothesis about the sources: their power spectra are all pair-wise nonproportional. Consequently, white noise sources as well as sources with identically shaped power-spectra cannot be separated. In AMUSE, this statistical hypothesis is captured by a one-lag covariance matrix. The efficiency of this method is increased considerably by utilizing covariance matrices with several lags, like in second-order blind identification (SOBI) [4], or Fourier cospectral matrices, like in AJDC [12]. We prefer the latter because (i) it is not easy to choose the appropriate set of lagged covariance matrices [75] [74], (ii) choosing the set of cospectral matrices, i.e., the number of frequencies, is directly related to the spectral characterization of searched sources [12], and (iii) AJDC has been shown to perform better than SOBI [19].

AMUSE is a BSS method separating sources with different power spectra, equivalent to an auto-correlation function. The solution is given by a generalized eigenvalue-eigenvector problem; thus the estimated sources are naturally sorted by order of auto-correlation. Typically, the two sources with the highest auto-correlation correspond to blinks and/or other eye-movements, and therefore can be removed without supervision [72]. Contrary to the previous approach, AMUSE does not require the operator to visually identify artifactual sources. A real-time implementation of this method computes covariance matrices on a $10 \mathrm{~s}$ memory to estimate the BSS separation matrix, and applies the BSS denoising on the last sample, or epoch, of data (same layout as in [48]).

AJDC is also a BSS method that separates sources with non-proportional power-spectra [12]. It is more powerful than AMUSE since the solution does not rely on a generalized eigenvalue-eigenvector solution, but on approximate joint diagonalization of several matrices. AJDC is still an unsupervised BSS technique, but it requires some processing to identify the artifactual sources to be eliminated. In order to do so, we apply AJDC to a 1 minute training signal that must contain eye blinks, and then we extract features from the obtained filters and sources. These features must be insensitive to source amplitude and sign (due to BSS uncertainties) and to the number of blinks contained in this training signal ${ }^{1}$. We extract (i) spatial features on the backward filters to capture: the left-right symmetry in signs and in amplitudes of coefficients, the strong power in frontal channels (ratio of amplitudes average between frontal and posterior areas), the typical frontal-to-posterior decaying

\footnotetext{
${ }^{1}$ This is why we do not used sources kurtosis [51] [66] [57] [16] which strongly depends on the number of blinks present in
} the considered signal. 
gradient (Spearman's rank correlation to measure the monotonic evolution from frontal to occipital channels); (ii) spectral features on estimated sources (ratio of averaged spectral powers between bands 1-3 Hz and 4-30 Hz), and (iii) the Pearson correlation between sources and most frontal channels. These features, independent from the EEG spatial configuration, will identify the spatial filter corresponding to the blink source. Finally, we monitor the blink source in real-time, using a thresholding criterion to decide which samples should be removed. It should be noted that the number of blinks (isolated or in quick succession) has no impact on this denoising procedure. Such a denoising procedure can be applied either online or offline, offering the possibility to include time offsets before and after the blink and thereby avoiding edge effects. If the time offset is null before the blink, the beginning of the blink can be missed, thus is not denoised, and can generate an edge between two consecutive EEG blocks.

\section{Comparison of denoising techniques}

\section{Power spectra computation}

We compare the average power spectrum obtained from each electrode using no denoising (raw data), manual denoising, and automatic denoising (by AMUSE and AJDC). The average power spectrum is computed on the four minutes of eyes open resting condition. Such an EEG signal contains eye blinks and is not influenced by external visual stimulation. The raw data is filtered with $4^{\text {th }}$-order Butterworth filters: $0.53 \mathrm{~Hz}$ high-pass filter, 50 $\mathrm{Hz}$ low-pass filter, and 55-65 Hz notch filter. To compute the power spectra, EEG signals are resampled at 128 $\mathrm{Hz}$ after anti-aliasing filtering. Overlapping epochs of 0.5 seconds are extracted every 0.25 seconds, then windowed (Hamming). From these windowed epochs, a power spectrum estimate is obtained via Fast Fourier Transform in the band-pass region $2-32 \mathrm{~Hz}$ with a frequency resolution of $2 \mathrm{~Hz}$. The average across the epochs is used to estimate the power spectra. The procedure we have just described corresponds to the well-known Welch method [88].

\section{ERP computation}

We compare the ERP obtained from each signal again using no denoising (raw data), manual denoising, and automatic denoising (AMUSE and AJDC). We focus on a time-domain analysis of the P100 ERP (a positive peak at $100 \mathrm{~ms}$ directly following a visual stimulation) because this response is more stable in time and displays less inter-individual variability as compared to later ERPs. EEG data is centered using a moving average of size 5 seconds (detrending). Then, the 100 ERP trials obtained in each condition are averaged. ERP enhancement is measured by computing the SNR. Since P100 is consistently distributed in parietal and occipital electrodes, the SNR is computed as the energy in channels P3, Pz, P4, O1, O2 on a window of 100 ms around the P100 peak divided by the total energy of the signal on a time window going from 0 to of $700 \mathrm{~ms}$ post-stimulus. We end up with 56 SNR values for each denoising method (4 conditions times 14 subjects).

\section{Statistical analysis}

In order to assess the difference between denoising procedures, the following statistical analyses are performed on the SNRs derived from the data. 


\section{Spectral analysis}

Initially, the spectra are averaged across all subjects and displayed in a topographic map that shows spectral content as a function of spatial position, allowing for visual interpretation of differences between two procedures. Then, for each subject, average spectra of both methods are compared using the Pearson correlation coefficients. Correlations are given by channels (computed along frequency dimension), and by frequency (computed along channel dimension). For inter-subject comparison, the median value and median absolute deviation of coefficients across subjects were used instead of mean and standard deviation, to account for asymmetric distributions. The median absolute deviation of a set $\left\{y_{i}\right\}_{i=1}^{I}$ is defined as median $_{j=1 \cdots I}\left\{\mid y_{j}-\right.$ $\left.\operatorname{median}_{i=1 \cdots I}\left\{y_{i}\right\} \mid\right\}$.

\section{ERP analysis}

For analysis purposes, SNRs were first log-transformed to promote symmetry of the distribution. A Wilcoxon signed-rank test was then used to test the null hypothesis that the difference between paired log-SNR values from the manually and automatically processed EEG records is equal to zero.

\section{Results of comparison between denoising techniques}

\section{Spectral analysis}

For the comparison between manual and AMUSE denoising, the spectrum at each electrode is displayed in Fig. 5 , averaged across all subjects. Spectra derived from raw signals are represented in green, manually denoised in black, and automatically denoised in blue. This convention for the line color will be kept henceforth. On frontal channels, spectral energy of the raw signals is removed by both denoising methods, which is related to blink removal. On parietal and occipital channels, differences between raw spectra and denoised spectra are reduced since there is less noise in these areas. We observe that AMUSE removes more energy than the manual denoising. Even in the absence of artifacts, due to its unsupervised nature, AMUSE tends to remove more energy than necessary, especially in low frequencies. This is a drawback of the AMUSE method and will be detailed later in the section "Limitations of AMUSE".

Spatial and spectral correlation coefficients are then computed to quantify the similarity between manual and automatic methods. Medians and median absolute deviations of correlation coefficients across subjects are plotted in Fig. 6, and median values are detailed in Table 1 and

Table 2. Regarding these results, we observe that spatial correlations are high, mainly situated between 0.98 and 1. On frontal channels, correlation is slightly lower, which can be explained by the fact that the automatic method always removes more energy than the manual one. We observe that spectral correlation values are lower than spatial correlation values, even if they remain quite high and show a consistent correlation at all frequencies between the two artifact-rejection methods. 


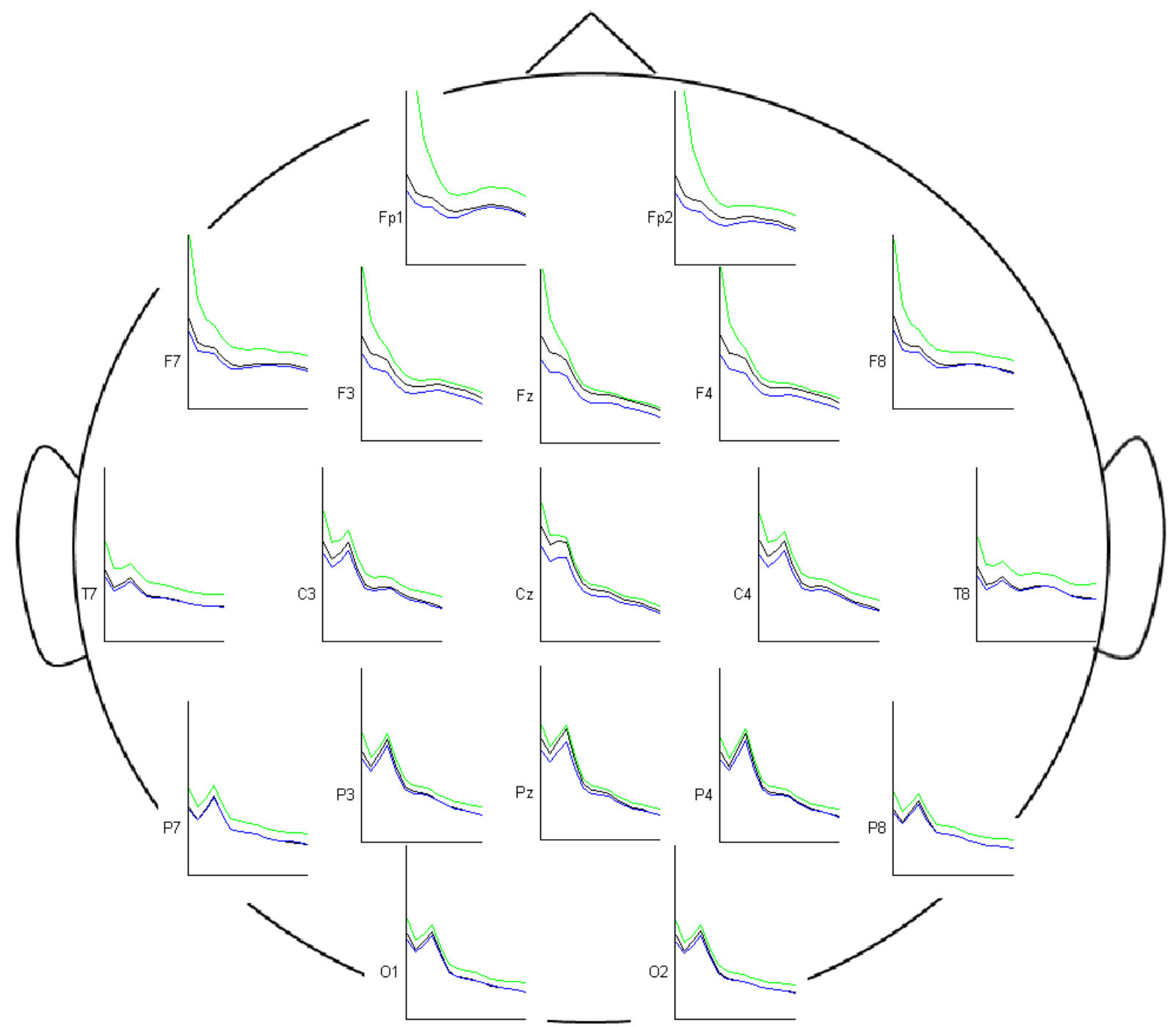

Fig. 5 Power spectra estimated at each electrode and averaged across all subjects, with frequencies on the $\mathrm{x}$-axis (given in $\mathrm{Hz}$ ) and spectral amplitudes on the y-axis. Spectra derived from raw signals are represented in green, those from manually denoised signals in black, and those from automatically denoised (by AMUSE) in blue. Manual denoising removes eye blinks in frontal regions, and it is close to raw data in posterior electrodes. Automatic denoising also removes eye blinks in frontal regions, but tends to remove more energy than necessary over the entire spectrum and in the majority of channels.

Table 1 Spatial correlation coefficients for each channel between spectra derived from manually and automatically (AMUSE) denoised signals; median across subjects.

\begin{tabular}{|c|c|c|c|c|c|c|c|c|c|}
\hline Channel & Fp1 & Fp2 & F7 & F3 & $\mathrm{Fz}$ & F4 & F8 & T3 & $\mathrm{C} 3$ \\
\hline Corr coeff & 0.929 & 0.938 & 0.992 & 0.990 & 0.994 & 0.991 & 0.987 & 0.994 & 0.996 \\
\hline$\overline{\mathrm{Cz}}$ & C4 & $\overline{\mathrm{T} 4}$ & T5 & P3 & $\mathrm{Pz}$ & P4 & F8 & O1 & $\mathrm{O} 2$ \\
\hline 0.998 & 0.996 & 0.993 & 0.998 & 0.998 & 0.999 & 0.999 & 0.997 & 0.999 & 0.999 \\
\hline
\end{tabular}

Table 2 Spectral correlation coefficients at each frequency (in $\mathrm{Hz}$ ) between spectra derived from manually and automatically (AMUSE) denoised signals; median across subjects.

\begin{tabular}{|c|c|c|c|c|c|c|c|c|c|}
\hline Frequency & 4 & 6 & 8 & 10 & 12 & 14 & 16 & 18 & 20 \\
\hline Corr coeff & 0.784 & 0.827 & 0.810 & 0.807 & 0.810 & 0.784 & 0.817 & 0.842 & 0.858 \\
\hline 22 & 24 & 26 & 28 & 30 & 32 & & & & \\
\hline 0.814 & 0.818 & 0.836 & 0.839 & 0.849 & 0.856 & & & & \\
\hline
\end{tabular}



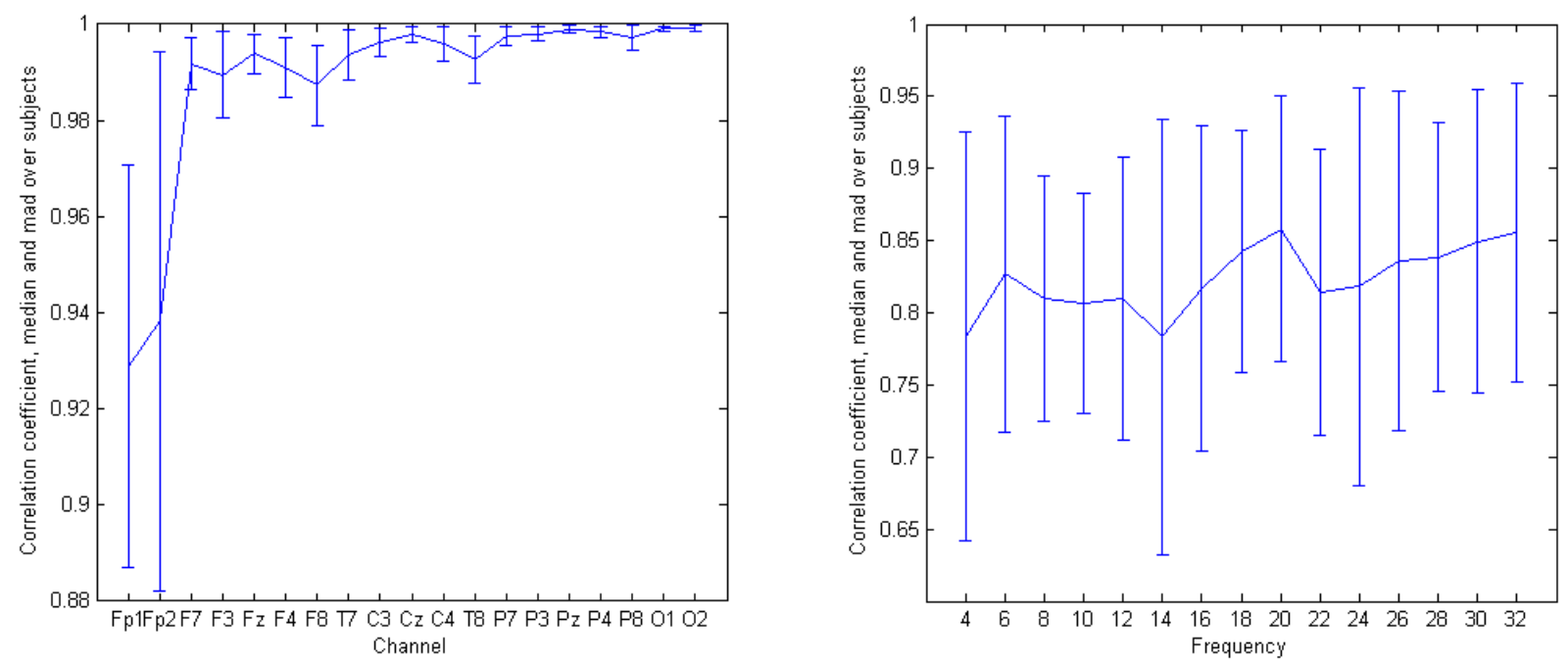

Fig. 6 Spatial (left) and spectral (right) correlation coefficients between spectra computed from manually and automatically (AMUSE) denoised EEG, giving medians and median absolute deviations across subjects.

For the comparison between manual and AJDC denoising, the spectrum at each electrode is displayed in Fig. 7, averaged across all subjects. Spectra derived from raw signals are represented in green, manually denoised in black, and automatically denoised in blue. On frontal channels, spectral energy of the raw signals has been removed by both denoising methods, which is related to blink removal. The quantity of removed energy is similar between denoising methods. On parietal and occipital channels, differences between raw spectra and denoised spectra are reduced since there is less noise in these areas.

Spatial and spectral correlation coefficients were computed to quantify the similarity between manual and automatic methods. Medians and median absolute deviations of correlation coefficients of subjects are plotted in Fig. 8 , and median values are detailed in

Table 3 and in

Table 4.

Regarding these results, we can observe that spatial correlations are high, mainly situated between 0.998 and 1 . On frontal channels, correlation is slightly lower because eye blinks are not removed by the same BSS method: Infomax for manual denoising and AJDC for automatic denoising (cf. the section "Description of denoising techniques"). Spectral correlations values are lower than spatial correlation values, even if both correlation values remain quite high. Low-frequencies have satisfactory correlations, contrary to the previous version of automatic denoising based on AMUSE, which removed blinks as well as low-frequency signal components (cf. the section "Limitations of AMUSE"). 


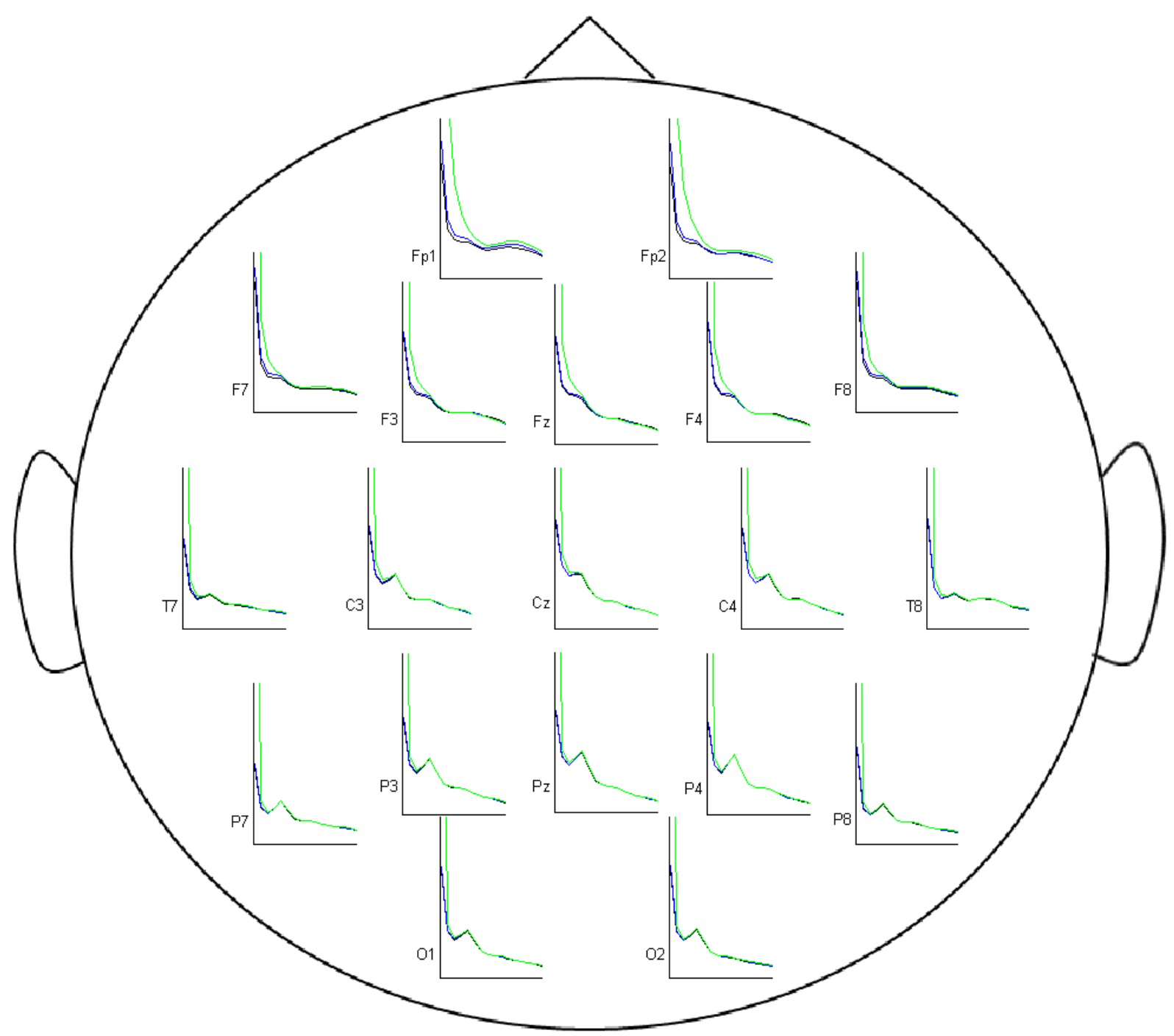

Fig. 7 Power spectra estimated at each electrode and averaged across all subjects, with frequencies on the $\mathrm{x}$-axis (given in $\mathrm{Hz}$ ) and spectral amplitudes on the y-axis. Spectra derived from raw signals are represented in green, those from manually denoised signals in black, and those from automatically denoised (by AJDC) in blue. Manual and automatic denoising methods have similar spectra: they remove eye blinks in frontal regions, and they are similar to raw data in other electrodes.

Table 3 Spatial correlation coefficients for each channel between spectra derived from manually and automatically (AJDC) denoised signals; median across subjects.

\begin{tabular}{|c|c|c|c|c|c|c|c|c|c|}
\hline Channel & Fp1 & Fp2 & F7 & F3 & $\mathrm{Fz}$ & F4 & F8 & T3 & $\mathrm{C} 3$ \\
\hline Corr coeff & 0.993 & 0.994 & 0.999 & 0.998 & 0.999 & 0.999 & 0.999 & 1.000 & 1.000 \\
\hline $\mathrm{Cz}$ & $\mathrm{C} 4$ & $\mathrm{~T} 4$ & T5 & P3 & $\mathrm{Pz}$ & $\mathrm{P} 4$ & F8 & O1 & $\mathrm{O} 2$ \\
\hline 1.000 & 1.000 & 1.000 & 1.000 & 1.000 & 1.000 & 1.000 & 1.000 & 1.000 & 1.000 \\
\hline
\end{tabular}

Table 4 Spectral correlation coefficients at each frequency (in $\mathrm{Hz}$ ) between spectra derived from manually and automatically (AJDC) denoised signals; median across subjects.

\begin{tabular}{|c|c|c|c|c|c|c|c|c|c|}
\hline Frequency & 2 & 4 & 6 & 8 & 10 & 12 & 14 & 16 & 18 \\
\hline Corr coeff & 0.925 & 0.906 & 0.939 & 0.939 & 0.953 & 0.937 & 0.926 & 0.937 & 0.939 \\
\hline 20 & 22 & 24 & 26 & 28 & 30 & 32 & & & \\
\hline 0.933 & 0.888 & 0.885 & 0.892 & 0.885 & 0.889 & 0.909 & & & \\
\hline
\end{tabular}



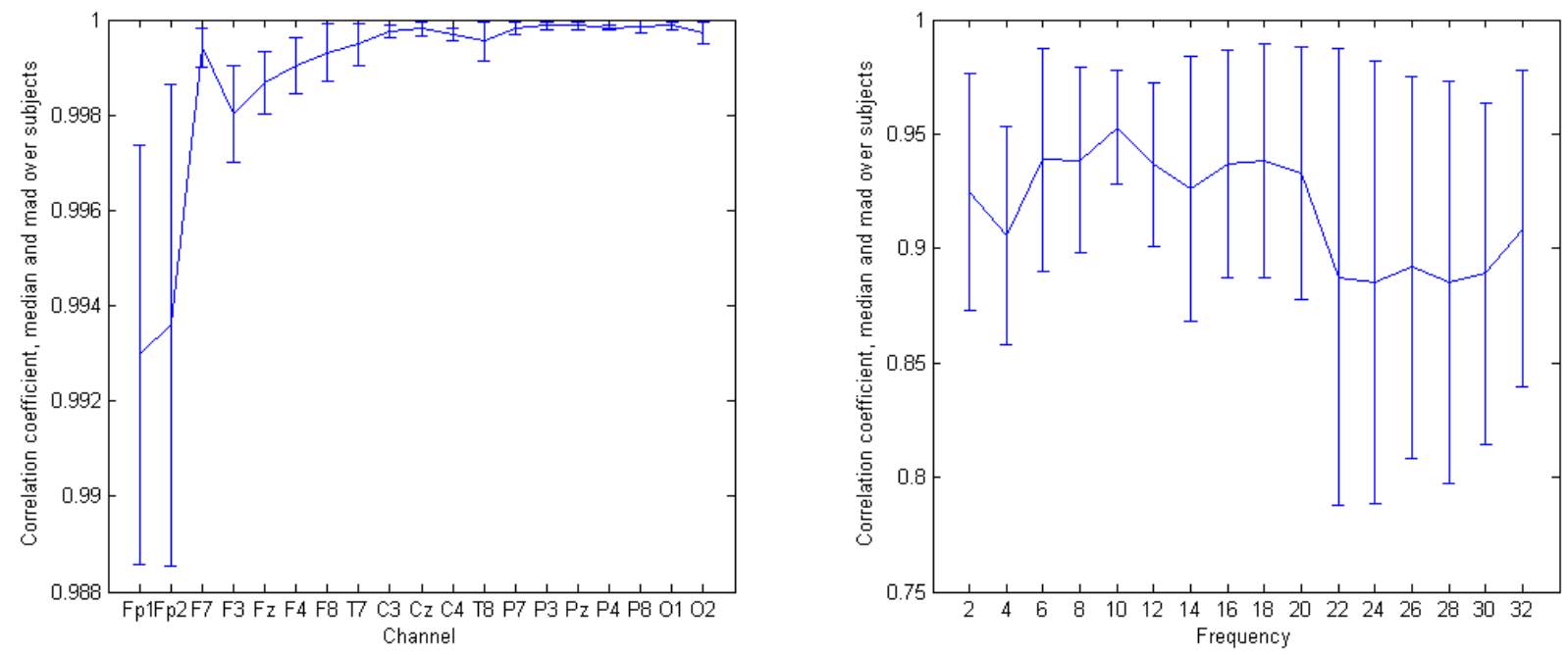

Fig. 8 Spatial (left) and spectral (right) correlation coefficients between spectra computed from manually and automatically (AJDC) denoised EEG, giving medians and median absolute deviations across subjects.

\section{ERP analysis}

For the AMUSE method, the grand averaged ERP is visualized at location O1 in Fig. 9 for the four conditions: Go, NoGo, Ignore and Novel. On each plot, time is indicated on the x-axis in samples and the amplitude of the ERP is indicated on the y-axis in $\mu \mathrm{V}$, with mean (line) and standard deviation (colored area) of ERP computed across subjects. ERPs extracted from raw signals are represented in green, manually denoised in black, and automatically denoised by AMUSE in blue. Due to space restriction, only the O1 electrode is shown, but it is representative of the event-related potentials observed in these data. We see that the distributions of cleaned ERP are quite similar in the four conditions. The inter-subject variability of the P100 estimation is lower for automatic denoising compared to the other methods.

We computed the SNR of the grand average ERPs. The advantage of the SNR criterion is that it takes into account the noise around the ERP; it is thus a good enhancement measure which does not require normalization of obtained ERPs. The logarithm transformation normalizes the distributions of SNR values. Using histograms, the distributions of log-SNR values for the manual and automatic (AMUSE) technique are plotted in Fig. 10. 

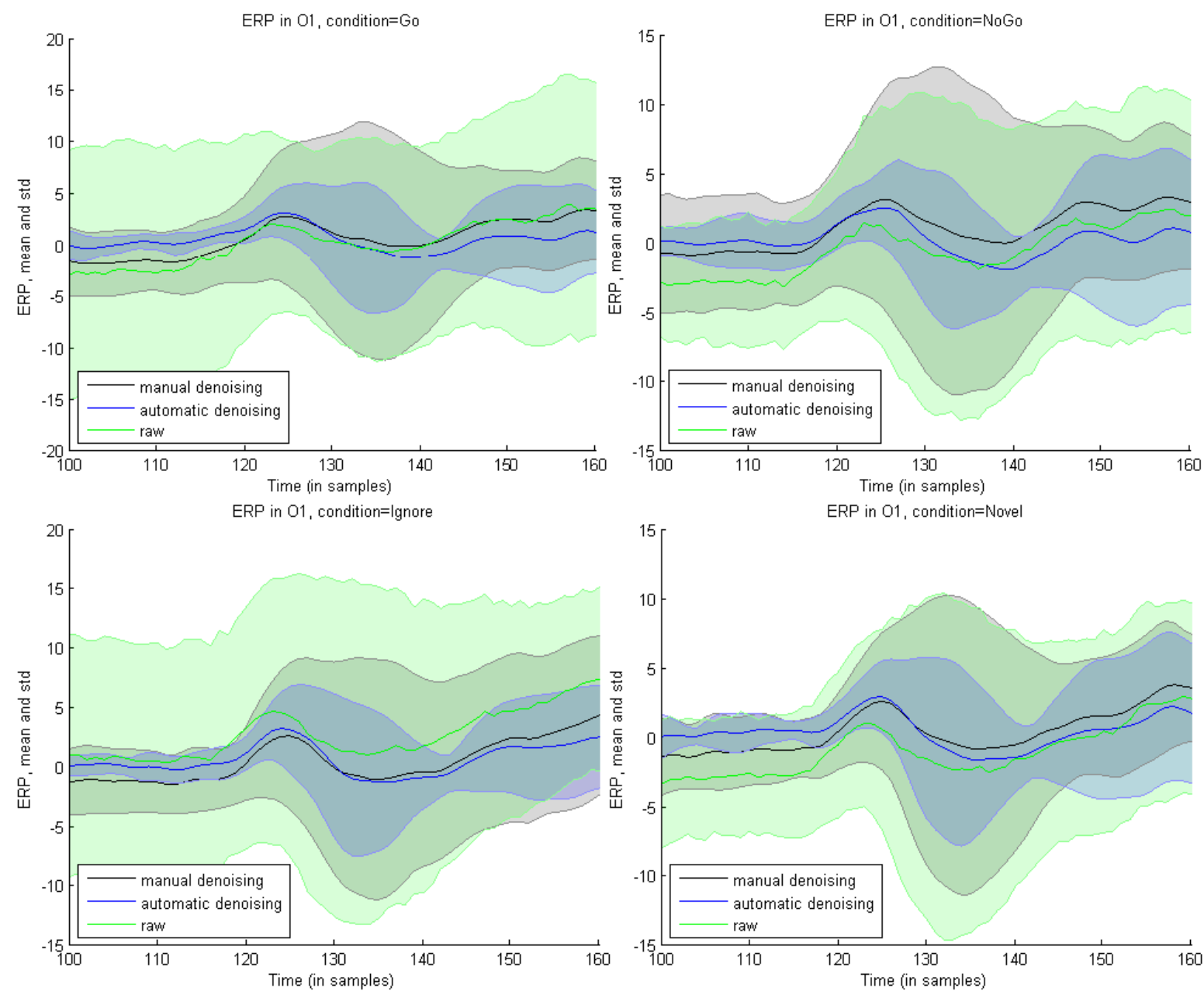

Fig. 9 Grand average ERP at location O1 for the conditions: Go (top left), NoGo (top right), Ignore (bottom left), Novel (bottom right). On each plot, time is indicated on the $\mathrm{x}$-axis in samples and the amplitude of the ERP is indicated on the yaxis in $\mu \mathrm{V}$, with mean (line) and standard deviation (colored area) of ERP computed across subjects. ERPs computed from raw signals are represented in green, manually denoised in black, and automatically denoised (by AMUSE) in blue.
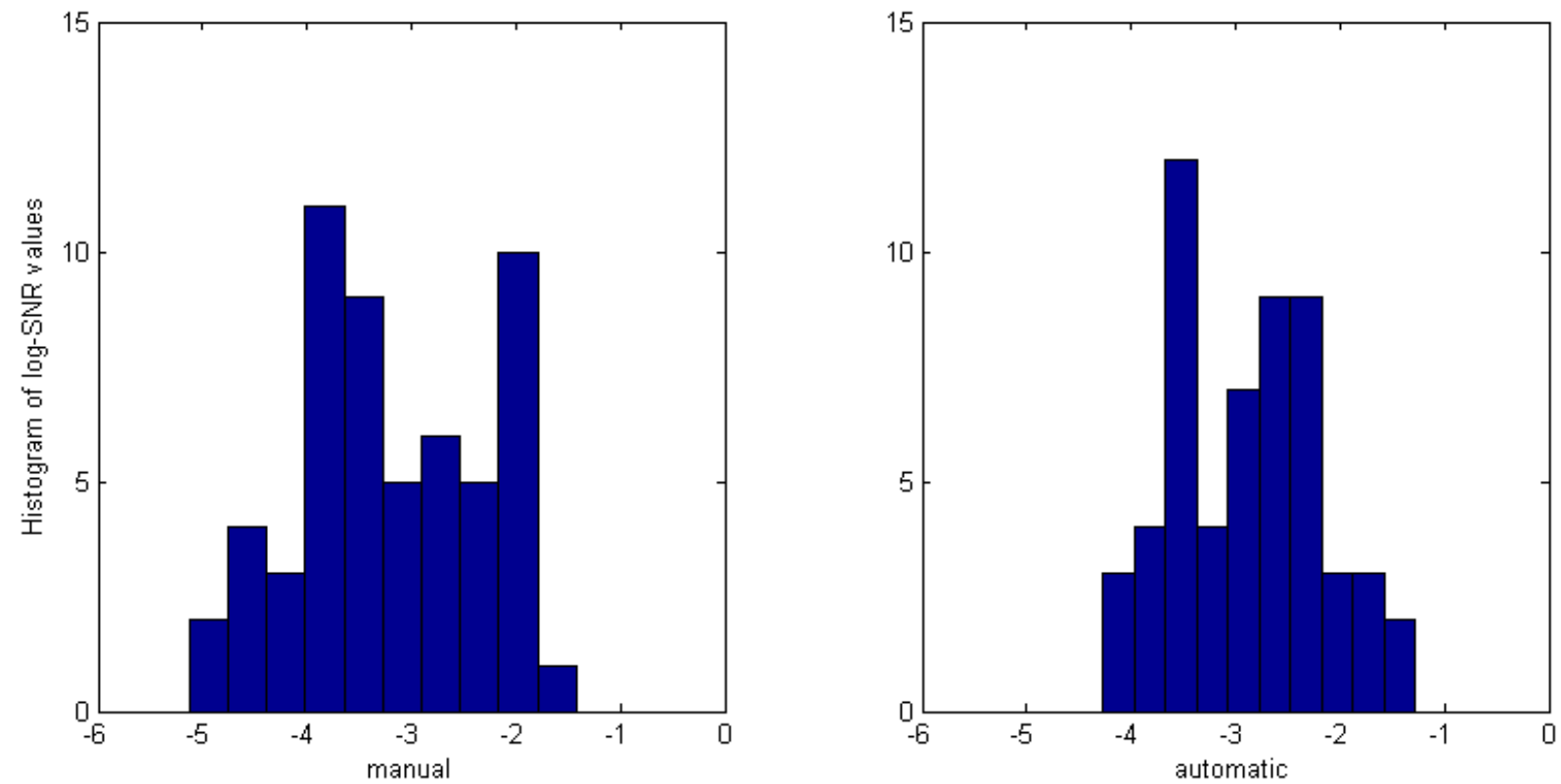

Fig. 10 Distribution of log-transformed SNR values for ERPs extracted from manually (left) and automatically by AMUSE (right) denoised EEG. 
In order to measure the similarity between these two distributions, the non-parametric Wilcoxon signed-rank test is applied on the log of the SNR values obtained with the two techniques. As reported in Table 5, the p-value is equal to 0.0151 , indicating that the observed difference between enhancements is statistically significant in favor of the automatic technique. Note that both techniques result in a significant enhancement as compared to the raw data.

Table 5 Details of statistical analysis comparing the distributions of log-SNR values of ERPs extracted from raw, manually denoised and automatically denoised (by AMUSE) signals. The values displayed are the mean and standard deviation of the log-SNR distributions; and distributions derived from manual and automated (AMUSE) are compared with a paired statistic.

\begin{tabular}{|l|c|c|c|}
\hline Methods & raw & manual & AMUSE \\
\hline Mean +/- sd of log SNR & $-4.44+/-1.25$ & $-3.20+/-0.91$ & $-2.90+/-0.69$ \\
\hline p-value & - & \multicolumn{2}{|c|}{0.0151} \\
\hline
\end{tabular}

Concerning the AJDC method, Fig. 11 shows the averaged ERP (in $\mu \mathrm{V}$ ) taken at electrode O1 for the four conditions: Go, NoGo, Ignore and Novel. On each plot, time is indicated on the $\mathrm{x}$-axis in samples and the amplitude of the ERP is indicated on the $y$-axis in $\mu \mathrm{V}$, with mean (line) and standard deviation (colored area) of ERP computed across subjects. ERPs extracted from raw signals are represented in green, manually denoised in black and automatically denoised by AJDC in blue. We can see that distributions of enhanced ERP are quite similar for the four conditions. Regarding the standard deviations, it can be noted that the subject variability of the P100 estimation is lower for automatic denoising compared to the other methods.

The SNR is computed from these averaged ERPs, as explained above. Using histograms, the distributions of logSNR values obtained with the manual and automatic (AJDC) technique are plotted in Fig. 12.

In order to test the null hypothesis that both denoising techniques provide equivalent SNR enhancements, the non-parametric Wilcoxon signed-rank test is applied on log SNR values obtained with both techniques. As reported in Table 6 , the p-value is equal to 0.3567 , indicating that the observed difference between the two denoising techniques is not statistically significant. Both techniques provide a significant enhancement with respect to the raw data. However, AJDC does not display the same limitations as displayed by AMUSE, as discussed in next section. 

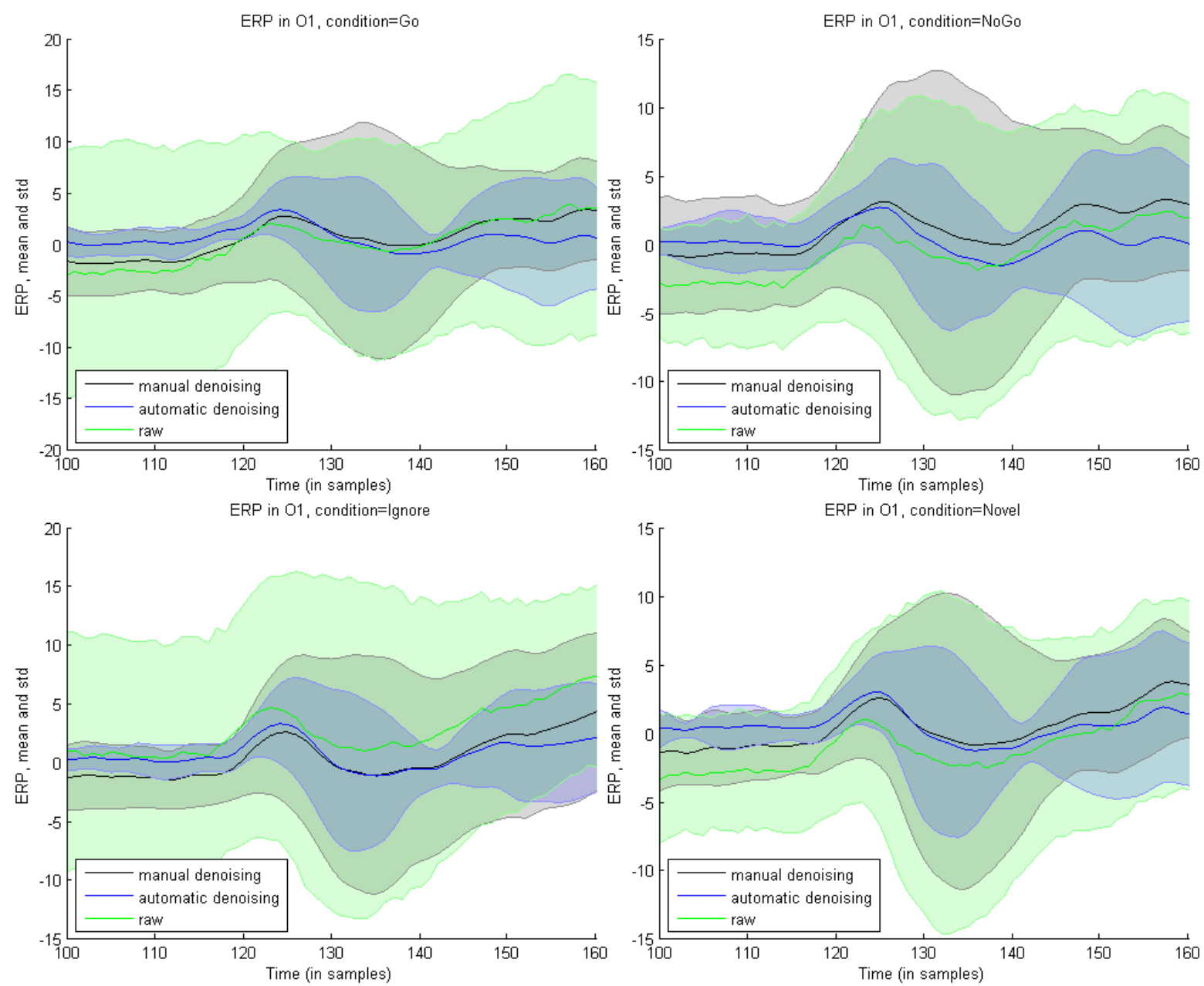

Fig. 11 Grand average ERP at location O1 for the conditions: Go (top left), NoGo (top right), Ignore (bottom left), Novel (bottom right). On each plot, time is indicated on the x-axis in samples and the amplitude of the ERP is indicated on the yaxis in $\mu \mathrm{V}$, with mean (line) and standard deviation (colored area) of ERP computed across subjects. ERPs computed from raw signals are represented in green, manually denoised in black, and automatically denoised (by AJDC) in blue.
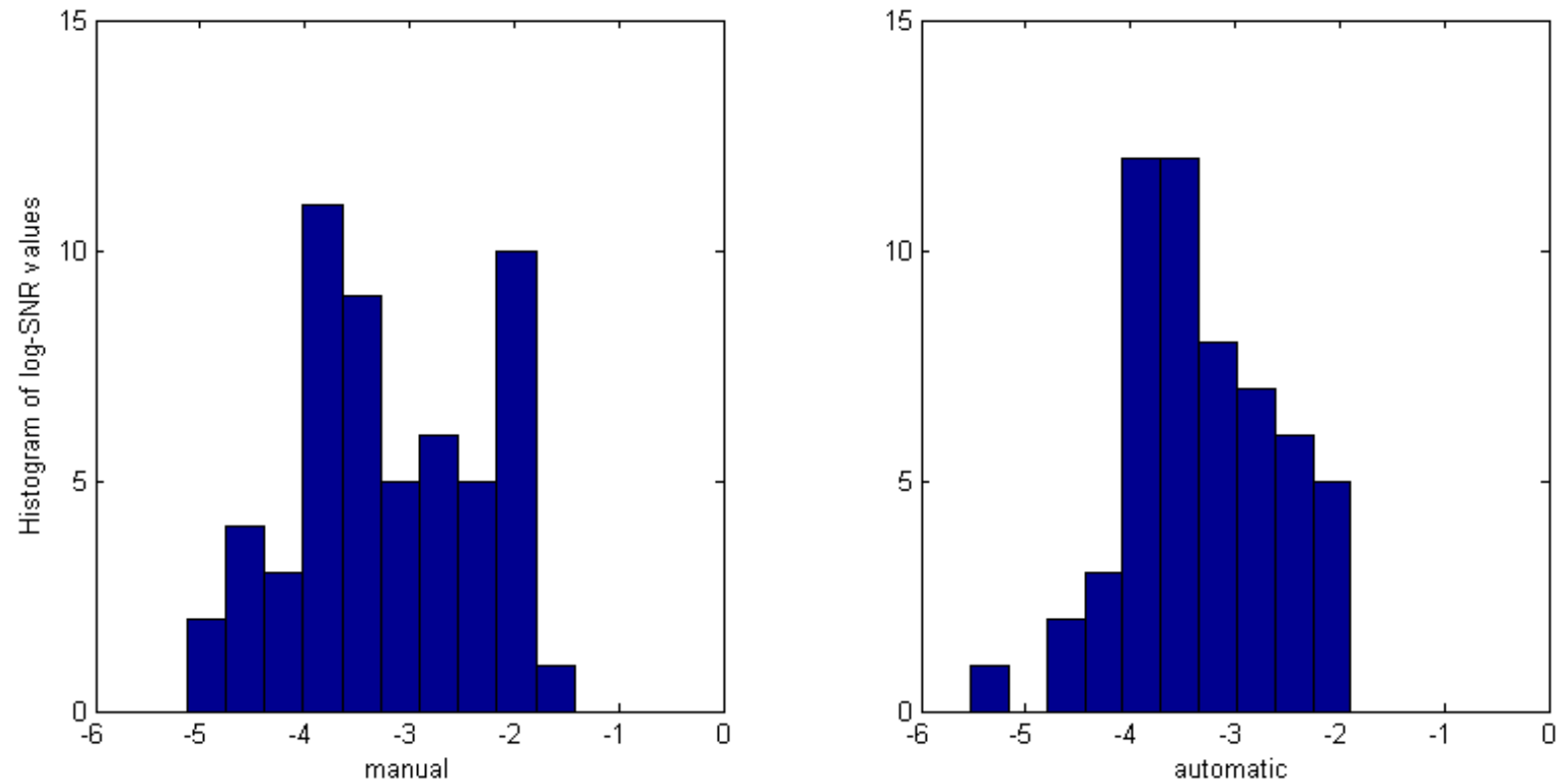

Fig. 12 Distribution of log-transformed SNR values for ERPs extracted from manually (left) and automatically by AJDC (right) denoised EEG. 
Table 6 Details of statistical analysis comparing the distributions of log-SNR values of ERPs extracted from raw, manually denoised and automatically denoised (by AJDC) signals. The values displayed are the mean and standard deviation of the log-SNR distributions; and distributions derived from manual and automated (AJDC) are compared with a paired statistic.

\begin{tabular}{|l|c|c|c|}
\hline Methods & raw & manual & AJDC \\
\hline Mean +/- sd of log SNR & $-4.44+/-1.25$ & $-3.20+/-0.91$ & $-3.32+/-0.75$ \\
\hline p-value & - & \multicolumn{2}{|c|}{0.3567} \\
\hline
\end{tabular}

\section{Limitations of AMUSE}

As observed in results, automatic denoising by AMUSE presents some drawbacks, which are detailed below.

\section{Block effects}

BSS methods for automatic denoising can produce block effects, the result of processing data online in small blocks $(6,25 \mathrm{~ms}$ for example, given by 8 samples at $128 \mathrm{~Hz})$. In eye blink removal, if the beginning of a blink wave is situated at the end of a data block, it can be missed and thus not denoised. Since the following block is denoised, it creates an edge between the two consecutive blocks.

\section{Delta band}

For eye blink removal, an auto-correlation based criterion is used to separate sources. Consequently, AMUSE tends to suppress the highest auto-correlated sources, i.e., sources composed mainly of low-frequencies. However, when EEG signals contain low-frequencies components as slow cortical potentials, they may be removed by AMUSE. To avoid this, the low cut-off frequency of the band-pass filter can be pulled-up, preventing the study of low-frequencies. Similarly, when there are no blinks in the signal, as in eyes closed recordings, the algorithm should not be activated, to avoid the removal of valuable information in the signal. This is illustrated in Fig. 13 showing the signal before (top) and after (middle) automatic blink removal when the low cut-off frequency of the band-pass filter is set to $0.5 \mathrm{~Hz}$. Sources estimated by the AMUSE method are also displayed (bottom). The blinks can be observed on the frontal channels of the filtered and denoised signals. This is due to the presence of the low-frequency components, which are captured by the BSS method as the two most auto-correlated sources, and are thus rejected. We see that the blink source appears as the third source, and is thus kept. Changing the low cut-off frequency of the band-pass filter from $0.5 \mathrm{~Hz}$ to $2 \mathrm{~Hz}$, as seen in Fig. 14 , moves the blink source to the last position, which is thus rejected from the denoised signal.

\section{Alpha band}

In Fig. 15, we illustrate how alpha components present in the original signal (top) can occasionally be rejected by automatic blink removal. We focus on the epoch between 1415s and 1416s, which contains strong alpha waves. In this epoch, since there is no blink, the most auto-correlated components of the signal are alpha components, as captured by the source separation (bottom). Since the AMUSE method rejects the two last sources (bottom), alpha components are rejected along with blinks, as observed in the denoised signal (middle). This phenomenon is amplified on signals without blinks, as for example signals in eyes closed sessions, which is why such an eye blink removal is not suitable in general for eyes-closed data. 


\section{Temporary signal contamination}

In Fig. 16, another example of undesirable behavior of AMUSE is presented. In the original signal (top), a transient wave appears at location $\mathrm{Cz}$ (channel 10) between 814s and 815s. As the analysis window of the AMUSE method is very long, it keeps the separation of artifacts in the frontal areas in memory. Consequently, this pattern, partially captured in the most auto-correlated sources, is removed from the signal as it is identified as a blink. This leads to the contamination of all the channels and introduces an additional block effect, which reduces the overall data quality on this segment. 

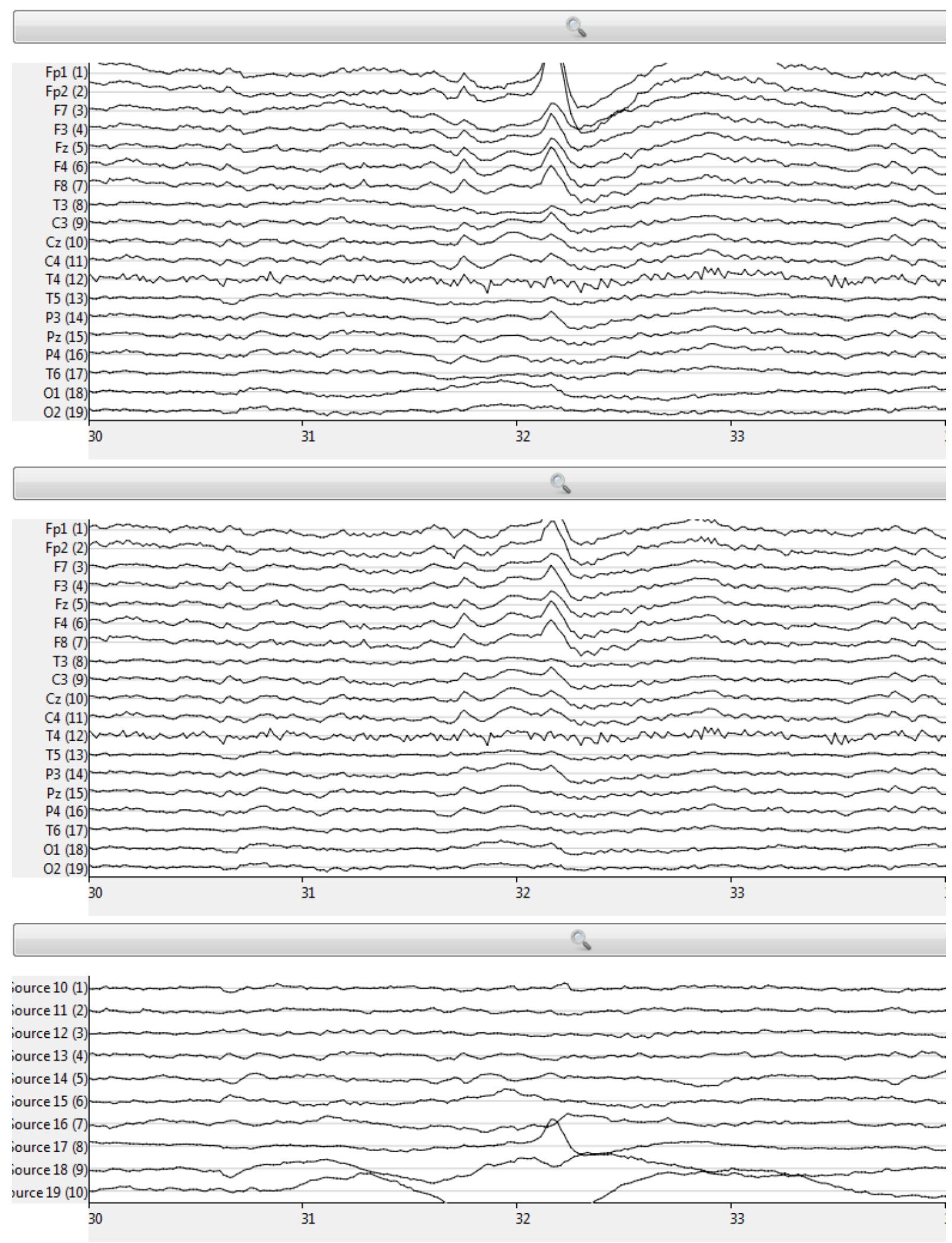

Fig. 13 Illustration of the eye blink removal with AMUSE with a low cut-off frequency of $0.5 \mathrm{~Hz}$, with the original signal (top), denoised signal (middle) and sources (bottom). The $\mathrm{x}$-axis shows the time in seconds, while the y-axis indicates the channel name (electrode or source number). In this example, one can see that the two most auto-correlated sources estimated by AMUSE (bottom plot, Sources 18 and 19) are low-frequency components, which are thus rejected. The blink source (Source 17) appears as the third most auto-correlated source, and is thus wrongly preserved. 

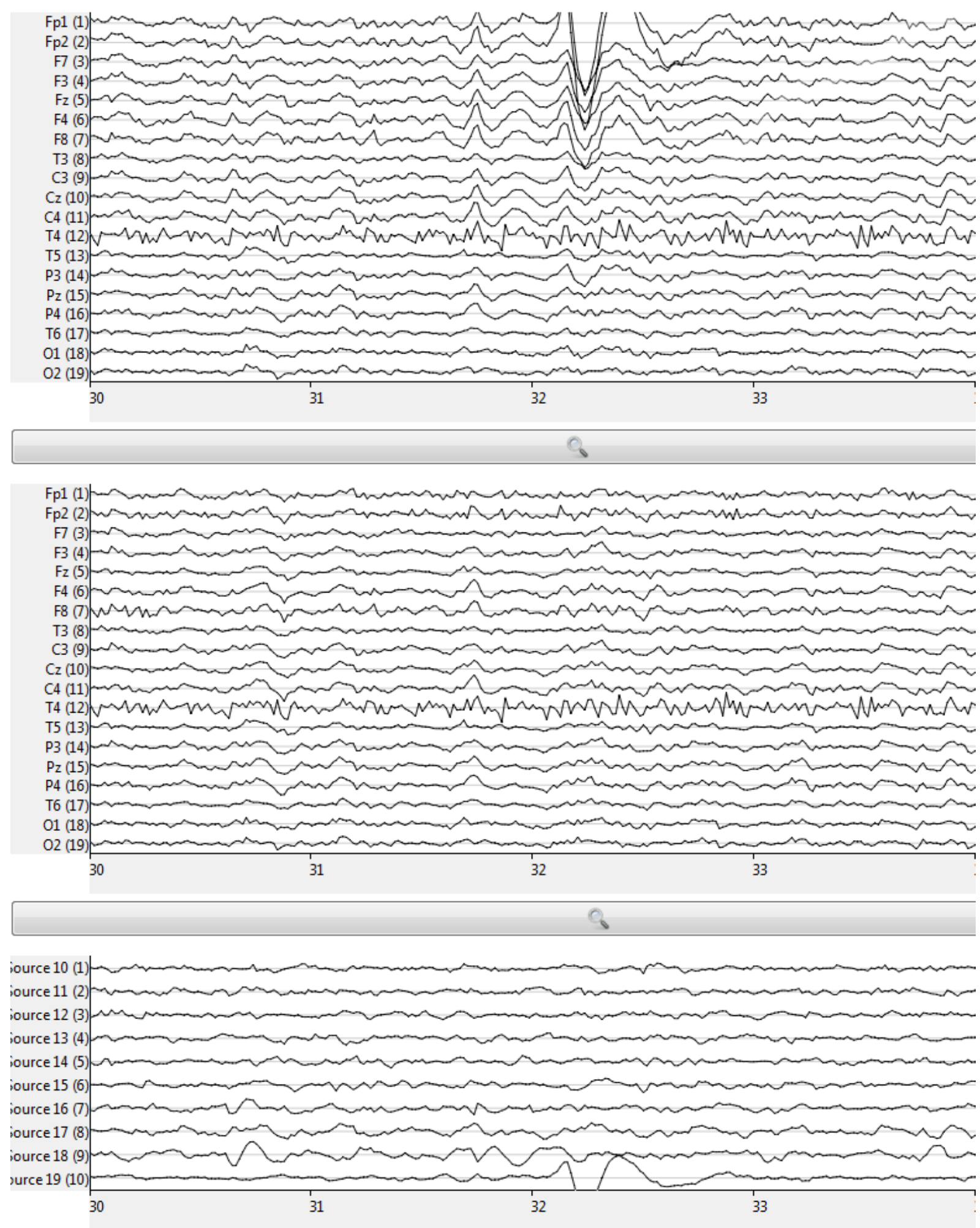

Fig. 14 Illustration of the eye blink removal with AMUSE with a low cut-off frequency of $2 \mathrm{~Hz}$, with the original signal (top), denoised signal (middle) and sources (bottom). The x-axis shows the time in seconds, while the y-axis indicates the channel name (electrode or source number). In this example, one can see that changing the low cut-off frequency of the bandpass filter from $0.5 \mathrm{~Hz}$ to $2 \mathrm{~Hz}$, moves the blink source to the last position, which is thus correctly rejected from the denoised signal. However, the delta band cannot be analyzed. 


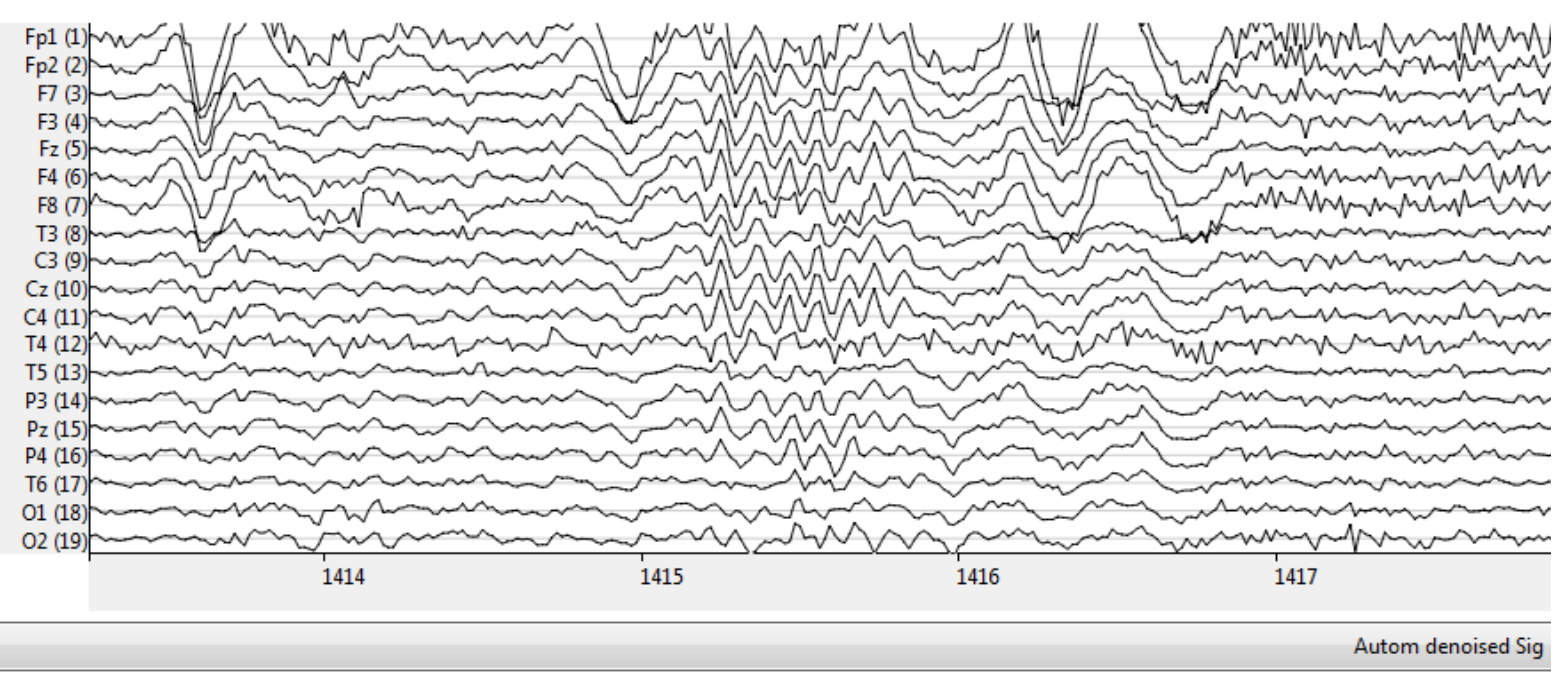

Fp1 (1) FP2 (2) (3) (3) F3 (3) (4) $\mathrm{Fz}_{2}(5)$ (1) F4 (6) (6) F8 (7) (8) (8) C3 (9) (8) $\mathrm{Cz}(10)$ (1) C4 (11) T4 (12) (n) T5 (13) (12) P3 (14) $\mathrm{Pz}$ (15) P4 (16) (15) T6 (17) (16) 01 (18) 02 (19)

iource 10 (1) iource11 (2) (1) iource 12 (3) iource13 (4) iource 14 (5) iource 15 (6) (6) iource 16 (7) iource18 (9) 10) (10) (14)

Fig. 15 Illustration of the eye blink removal with AMUSE on the signal containing alpha waves, with the original signal (top), denoised signal (middle) and sources (bottom). The $\mathrm{x}$-axis shows the time in seconds, while the $\mathrm{y}$-axis indicates the channel name (electrode or source number). In this example, since there is no blink between 1415s and 1416s, the most autocorrelated components of the signal are alpha waves, which are incorrectly rejected along with blinks. 


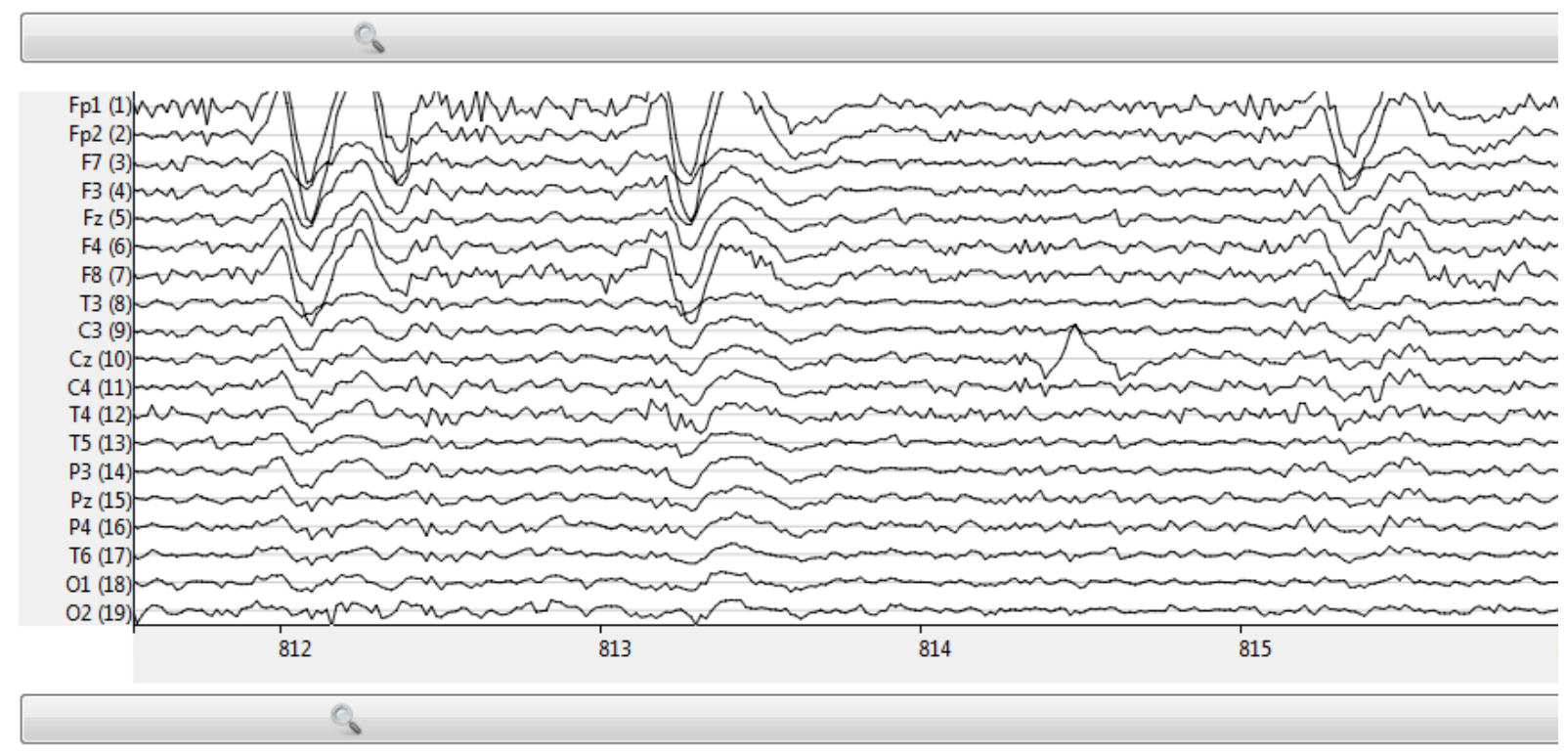

Fp1 (1) (1)

F22 (2) (3)

F3 (4) (3) (5)

$\mathrm{Fz}(5)$ (5)

F4 (6) (6)

F8 (7) (7)

T3 (8)

C3 (9) $\mathrm{Cz}(10)$ (1) C4 (11) T4 (12) (1) P3 (14) (13) $P_{2}(15)$ P4 (16) T6 (17) 01 (18) 02 (19) 812 813 814 815

Q

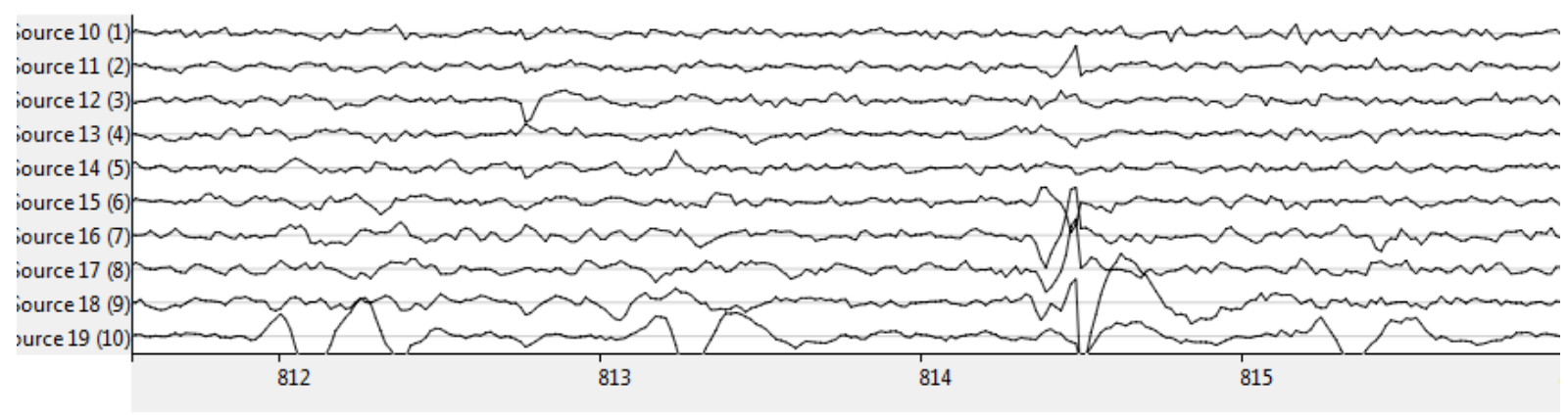

Fig. 16 Illustration of the eye blink removal with AMUSE on the signal with a small artifactual wave, with the original signal (top), denoised signal (middle) and sources (bottom). The $\mathrm{x}$-axis shows the time in seconds, while the y-axis indicates the channel name (electrode or source number). In this example, the transient wave in $\mathrm{Cz}$ between $814 \mathrm{~s}$ and $815 \mathrm{~s}$ is partly captured in the most auto-correlated sources of AMUSE and is removed from the signal as if it was a blink, leading to the contamination of all channels. 


\section{Discussion}

Arguably, manual and careful visualization of the EEG data with a source separation technique is the gold standard for cleaning artifacts from EEG data. However, such a technique comes with significant drawbacks. Primarily, the technique is highly operator-dependent and introduces intra- and inter-individual variability that hinders the quality and the repeatability of research using such a technique [57] [51] [15] [39] [27]. The same database processed by the same operator at different days, or by two operators, may provide different results, which leads to great variability in the processing quality. For instance, in the database we report on, manual inconsistencies were observed in the manual processing of subject 3 , leading to the exclusion of this record from the study.

Automatic denoising methods also present occasional drawbacks. Limitations of AMUSE denoising have been discussed in the section "Limitations of AMUSE". AJDC denoising addresses these limitations; however, it requires a calibration step over 1 min of signal (cf. the section "Online unsupervised denoising”), selected as being representative of the artifacts. Ideally, this training signal should contain only eye blink artifacts. To actually be unsupervised, this algorithm must extract several spatial and spectral features in order to robustly identify the blink source. Too many artifacts or too few blinks during this training signal can lead to a bad sources estimation and difficulty with blink source selection.

While both manual and automatic techniques have drawbacks, their objective comparison carried out in this study reveals that they result in comparable spectral and ERP features. Remark that the AJDC method used in this article can also be applied to magnetoencephalographic (MEG) signals, since many BSS methods have been applied to EEG as well to MEG [31] [2] [28] [58].

Results obtained in this study are consistent with comparative studies [34] [36] [64] [65] [82] [60], showing that spectral coloration of SOS based BSS methods stand out as the best performing approach for separating and removing eye-blink artifacts from EEG, compared to the mutual independence of HOS methods. SOS methods have other advantages: they are more robust to outliers and thus require less data [34] [78] [64] [12], they make no hypothesis on sources distribution, such as non-Gaussianity, and spectral coloration seems to be an appropriate criterion for spontaneous and induced EEG [78] [12]. That is why it is important to avoid confusion between methods. For instance, SOBI is often considered as belonging to the ICA family [59] [91] [18] [82] [48] [81] [60], but it is not. Consequently, instead of the question "ICA or not?" [77] [52] [17], a more pertinent question would be "how appropriate are the criteria used to separate EEG sources by BSS?"

\section{Conclusion}

This article compares two denoising techniques: the online automatic denoising methods, AMUSE and AJDC, and manual denoising. Two sets of measures are used to compare the performance of these denoising techniques on real data. Our analysis shows that, compared to AMUSE, AJDC gives results that are more comparable to those obtained by a manual denoising procedure. For power spectra comparison, we showed that the automatic AJDC technique is very similar to the manual one in that both techniques remove a similar quantity of energy in all regions, which is an improvement to the AMUSE method. Consequently, this method can be used for protocols in low-frequency bands, such as the theta and delta bands. Likewise, the ERP analysis shows that important ERP characteristics are preserved after denoising by AJDC, and there is no statistically significant 
difference between manual and automatic SNR enhancements, while both techniques significantly enhanced results when compared to the absence of processing.

Careful manual review of the signal should, in theory, always provide as good results as a good online technique. We argue, however, that in practice, manual review comes with limitations (quality, reproducibility, and cost) which are difficult to circumvent. On the other hand, the automated method based on AJDC also comes with limitations (training signal with "representative" data), but offers reproducibility and speed. Automated analysis is particularly convenient for quantitative EEG studies (qEEG) where all records should be processed by the same denoising pipeline before undergoing further analysis. Given the gain in speed and quality offered by the AJDC when compared to the manual review of signals and the resulting improvement in the speed/quality tradeoff, this automated approach proves feasible and convenient.

In conclusion, AJDC is an unsupervised online technique convenient for real-time brain monitoring applications such as NFB and BCI. As the denoising procedure maximizes speed, it minimizes the feedback delay and thus provides responsive applications.

\section{Conflict of interest}

Q. Barthélemy, L. Mayaud and Y. Renard work at Mensia Technologies and own stocks in the company. D. Kim and S. Kang work at Seoul National University. J. Gunkelman works at Brain Science International. M. Congedo works at CNRS (Centre National de la Recherche Scientifique), Grenoble-Alpes University, Grenoble University of Technology, and is scientific advisor at Mensia Technologies.

\section{Acknowledgement}

The authors would like to thank J.-P. Lefaucheur and anonymous reviewers for their fruitful comments, and S. Collin for her help about English usage.

\section{References}

[1] Arns M, de Ridder S, Strehl U, Breteler M, Coenen A. Efficacy of neurofeedback treatment in ADHD: The effects on inattention, impulsivity and hyperactivity: A meta-analysis. Clin EEG Neurosci 2009;40:180-9.

[2] Barbati G, Porcaro C, Zappasodi F, Rossini P, Tecchio F. Optimization of an independent component analysis approach for artifact identification and removal in magnetoencephalographic signals. Clin Neurophysiol 2004;115:1220-32.

[3] Bell AJ, Sejnowski TJ. An information-maximization approach to blind separation and blind deconvolution. Neural Comput 1995;7:1129-59.

[4] Belouchrani A, Abed-Meraim K, Cardoso J, Moulines E. A blind source separation technique using second-order statistics. IEEE Trans Signal Process 1997;45:434-44.

[5] Bigdely-Shamlo N, Kreutz-Delgado K, Kothe C, Makeig S. Eyecatch: Data-mining over half a million EEG independent components to construct a fully-automated eye-component detector. In Int. Conf. IEEE Engineering in Medicine and Biology Society (EMBC), 2013.

[6] Boudet S, Peyrodie L, Gallois P, Vasseur C. Filtering by optimal projection and application to automatic artifact removal from EEG. Signal Processing 2007;87:1978-92. 
[7] Cardoso JF, Souloumiac A. Blind beamforming for non-Gaussian signals. IEE Proceedings F - Radar and Signal Processing 1993;140:362-70.

[8] Chaumon M, Bishop DVM, Busch NA. A practical guide to the selection of independent components of the electroencephalogram for artifact correction. J Neurosci Methods 2015;250:47-63.

[9] Cichocki A, Amari S. Adaptive Blind Signal and Image Processing : Learning Algorithms and Applications. John Wiley \& Sons, 2003.

[10] Comon P. Independent Component Analysis, a new concept? Signal Processing 1994;36:287-314.

[11] Comon P, Jutten C. Handbook of Blind Source Separation, Independent Component Analysis and Applications. New York: Academic, 2010.

[12] Congedo M, Gouy-Pailler C, Jutten C. On the blind source separation of human electroencephalogram by approximate joint diagonalization of second order statistics. Clin Neurophysiol 2008;119:2677-86.

[13] Croft RJ, Chandler J, Barry RJ, Cooper N, Clarke A. EOG correction: A comparison of four methods. Psychophysiology 2005;42:16-24.

[14] Croft RJ, Barry RJ. Removal of ocular artifact from the EEG: a review. Clin Neurophysiol 2000;30:519.

[15] Daly I, Nicolaou N, Nasuto S, Warwick K. Automated artifact removal from the electroencephalogram: A comparative study. Clin EEG Neurosci 2013;44:291-306.

[16] Daly I, Scherer R, Billinger M, Müller-Putz G. FORCe: Fully online and automated artifact removal for brain-computer interfacing. IEEE Trans Neural Syst Rehabil Eng 2015;23:725-36.

[17] Delorme A. ICA phase distortion, https://sccn.ucsd.edu/wiki/ica_phase distortion, 2017.

[18] Delorme A, Sejnowski T, Makeig S. Enhanced detection of artifacts in EEG data using higher-order statistics and independent component analysis. NeuroImage 2007;34:1443-9.

[19] Doron E, Yeredor A. Asymptotically optimal blind separation of parametric Gaussian sources. In Int. Conf. on Independent Component Analysis and Signal Separation, p. 390-7, 2004.

[20] Fatourechi M, Bashashati A, Ward RK, Gary GE. EMG and EOG artifacts in brain computer interface systems: A survey. Clin Neurophysiol 2007;118:480-94.

[21] Flexer A, Bauer H, Pripfl J, Dorffner G. Using ICA for removal of ocular artifacts in EEG recorded from blind subjects. Neural Networks 2005;18:998-1005.

[22] Gasser T, Sroka L, Möcks J. The transfer of EOG activity into the EEG for eyes open and closed. Electroencephalogr Clin Neurophysiol 1985;61:181-93.

[23] Ghandeharion H, Erfanian A. A fully automatic ocular artifact suppression from EEG data using higher order statistics: Improved performance by wavelet analysis. Med Eng Phys 2010;32:720-9.

[24] Gratton G. Dealing with artifacts: The EOG contamination of the event-related brain potential. Behav Res Methods Instrum Comput 1998;30:44-53.

[25] Gratton G, Coles MGH, Donchin E. A new method for off-line removal of ocular artifact. Electroencephalogr Clin Neurophysiol 1983;55:468-84.

[26] Hagemann D, Naumann E. The effects of ocular artifacts on (lateralized) broadband power in the EEG. Clin Neurophysiol 2001;112:215-31.

[27] Hartmann M, Schindler K, Gebbink T, Gritsch G, Kluge T. PureEEG: Automatic EEG artifact removal for epilepsy monitoring. Neurophysiol Clin 2014;44:479-90. 
[28] Hesse C, James C. On semi-blind source separation using spatial constraints with applications in EEG analysis. IEEE Trans Biomed Eng 2006;53:2525-34.

[29] Islam MK, Rastegarnia A, Yang Z. Methods for artifact detection and removal from scalp EEG: A review. Neurophysiol Clin 2016;46:287-305.

[30] Iwasaki M, Kellinghaus C, Alexopoulos AV, Burgess RC, Kumar AN, Han YH, et al. Effects of eyelid closure, blinks, and eye movements on the electroencephalogram. Clin Neurophysiol 2005;116:878-85.

[31] James CJ, Gibson, OJ. Temporally constrained ICA: An application to artifact rejection in electromagnetic brain signal analysis. IEEE Trans Biomed Eng 2003;50:1108-16.

[32] Jas M, Engemann DA, Bekhti Y, Raimondo F, Gramfort A. Autoreject: Automated artifact rejection for MEG and EEG data. NeuroImage 2017;159:417-29.

[33] Jasper HH. The ten-twenty electrode system of the International Federation. Electroencephalogr Clin Neurophysiol 1958;10:371-5.

[34] Joyce CA, Gorodnitsky IF, Kutas M. Automatic removal of eye movement and blink artifacts from EEG data using blind component separation. Psychophysiology 2004;41:313-25.

[35] Jung TP, Makeig S, Humphries C, Lee TW, McKeown MJ, Iragui V, Sejnowski TJ. Removing electroencephalographic artifacts by blind source separation. Psychophysiology 2000;37:163-78.

[36] Kierkels J, van Boxtel G, Votgen L. A model-based objective evaluation of eye movement correction in EEG recordings. IEEE Trans Biomed Eng 2006;53:246-53.

[37] Kilicarslan A, Grossman RG, Contreras-Vidal JL. A robust adaptive denoising framework for real-time artifact removal in scalp EEG measurements. J Neural Eng 2006;13:026013.

[38] Klados MA, Papadelis C, Braun C, Bamidis PD. REG-ICA: A hybrid methodology combining blind source separation and regression techniques for the rejection of ocular artifacts. Biomed Signal Process Control 2011;6:291-300.

[39] Lawhern V, Hairston WD, Robbins K. DETECT: a MATLAB toolbox for event detection and identification in time series, with applications to artifact detection in EEG signals. PLoS One 2013;8:1-13.

[40] Le Van P, Urrestarazu E, Gotman J. A system for automatic artifact removal in ictal scalp EEG based on independent component analysis and bayesian classification. Clin Neurophysiol 2006;117:912-27.

[41] Lee TW, Girolami M, Sejnowski TJ. Independent component analysis using an extended infomax algorithm for mixed sub-Gaussian and super-Gaussian sources. Neural Comput 1999;11:417-41.

[42] Li Y, Ma Z, Lu W, Li Y. Automatic removal of the eye blink artifact from EEG using an ICA-based template matching approach. Physiol Meas 2006;27:425-36.

[43] Lins OG, Piction TW, Berg P, Scherg M. Ocular artifacts in EEG and event-related potentials I: Scalp topography. Brain Topogr 1993;6:51-63.

[44] Lins OG, Piction TW, Berg P, Scherg M. Ocular artifacts in recording EEGs and event-related potentials II: Source dipoles and source components. Brain Topogr 1993;6:65-78.

[45] Lotte F, Bougrain L, Clerc M. Electroencephalography (EEG)-based Brain-Computer Interfaces. Wiley Encyclopedia of Electrical and Electronics Engineering, 2015.

[46] Manoilov P. EEG eye-blinking artefacts power spectrum analysis. In Int. Conf. Computer Systems and Technologies - CompSysTech’06, 2006. 
[47] Manoilov P. Eye-blinking artefacts analysis. In Int. Conf. Computer Systems and Technologies CompSysTech'07, 2007.

[48] Mayeli A, Zotev V, Refai H, Bodurka J. Real-time EEG artifact correction during fMRI using ICA. J Neurosci Methods 2016;274:27-37.

[49] McFarland DJ, Sarnacki WA, Vaughan TM, Wolpaw JR. Brain-computer interface (BCI) operation: signal and noise during early training sessions. Clin Neurophysiol 2005;116:56-62.

[50] Mennes M, Wouters H, Vanrumste B, Lagae L. Validation of ICA as a tool to remove eye movement artifacts from EEG/ERP. Psychophysiology 2010;47:1142-50.

[51] Mognon A, Jovicich J, Bruzzone L, Buiatti M. ADJUST: An automatic EEG artifact detector based on the joint use of spatial and temporal features. Psychophysiology 2011;48:229-40.

[52] Montefusco-Siegmund R, Maldonado PE, Devia C. Effects of ocular artifact removal through ICA decomposition on EEG phase. In IEEE/EMBS Int. Conf. on Neural Engineering (NER), p. 1374-7, 2013.

[53] Montgomery DD. Change: Detection and modification. Appl Psychophysiol Biofeedback 2001;26:21526.

[54] Moretti DV, Babiloni F, Carducci F, Cincotti F, Remondini E, Rossini PM, et al. Computerized processing of EEG-EOG-EMG artifacts for multi-centric studies in EEG oscillations and event-related potentials. Int J Psychophysiol 2003;47:199-216.

[55] Nazarpour K, Mohseni H, Hesse C, Chambers J, Sanei S. A novel semiblind signal extraction approach for the removal of eye-blink artifact from EEGs. EURASIP J Adv Signal Process 2008;2008:98.

[56] Nicolaou N, Nasuto SJ. Automatic artefact removal from event-related potentials via clustering. J VLSI Signal Process Syst Signal Image Video Technol 2007;48:173-83.

[57] Nolan H, Whelan R, Reilly RB. FASTER: Fully automated statistical thresholding for EEG artifact rejection. J Neurosci Methods 2010;192:152-62.

[58] Okada Y, Jung J, Kobayashi T. An automatic identification and removal method for eye-blink artifacts in event-related magnetoencephalographic measurements. Physiol Meas 2007;28:1523.

[59] Onton J, Westerfield M, Townsend J, Makeig S. Imaging human EEG dynamics using independent component analysis. Neurosci Biobehav Rev 2006;30:808-22.

[60] Pontifex MB, Gwizdala KL, Parks AC, Billiner M, Brunner C. Variability of ICA decomposition may impact EEG signals when used to remove eyeblink artifacts. Psychophysiology 2017;54:386-98.

[61] Pontifex MB, Miskovic V, Laszlo S. Evaluating the efficacy of fully automated approaches for the selection of eyeblink ICA components. Psychophysiology 2017;54:780-91.

[62] Renard Y, Lotte L, Gibert G, Congedo M, Maby E, Delannoy V, Bertrand O, Lécuyer A. OpenViBE: An open-source software platform to design, test, and use brain-computer interfaces in real and virtual environments. Presence 2010;19:35-53.

[63] Riccio CA, Reynolds CR, Lowe P, Moore JJ. The continuous performance test: a window on the neural substrates for attention? Arch Clin Neuropsychol 2002;17:235-72.

[64] Romero S, Mananas MA, Barbanoj MJ. A comparative study of automatic techniques for ocular artifact reduction in spontaneous EEG signals based on clinical target variables: A simulation case. Comput Biol Med 2008;38:348-60. 
[65] Romero S, Mananas MA, Barbanoj MJ. Ocular reduction in EEG signals based on adaptive filtering, regression and blind source separation. Ann Biomed Eng 2009;37:176-91.

[66] Roy RN, Charbonnier S, Bonnet S. Eye blink characterization from frontal EEG electrodes using source separation and pattern recognition algorithms. Biomed Signal Process Control 2014;14:256-64.

[67] Sagha H, Perdikis S, del R. Millán J, Chavarriaga R. Quantifying electrode reliability during braincomputer interface operation. IEEE Trans Biomed Eng 2015;62:858-64.

[68] Sameni R, Gouy-Pailler C. An iterative subspace denoising algorithm for removing electroencephalogram ocular artifacts. J Neurosci Methods 2014;225:97-105.

[69] Schlögl A, Keinrath C, Zimmermann D, Scherer R, Leeb R, Pfurtscheller G. A fully automated correction method of EOG artifacts in EEG recordings. Clin Neurophysiol 2007;118:98-104.

[70] Shao SY, Shen KQ, Ong CJ, Wilder-Smith EPV, Li XP. Automatic EEG artifact removal: A weighted support vector machine approach with error correction. IEEE Trans Biomed Eng 2009;56:336-44.

[71] Shoker L, Sanei S, Chambers J. Artifact removal from electroencephalograms using a hybrid BSS-SVM algorithm. IEEE Signal Process Lett 2005;12:721-24.

[72] Sun L, Rieger J, Hinrichs H. Maximum noise fraction (MNF) transformation to remove ballistocardiographic artifacts in EEG signals recorded during fMRI scanning. NeuroImage 2009;46:144-53.

[73] Sweeney KT, Ward TE, McLoone SF. Artifact removal in physiological signals - practices and possibilities. IEEE Trans Inf Technol Biomed 2012;16:488-500.

[74] Tang AC, Liu JY, Sutherland MT. Recovery of correlated neuronal sources from EEG: The good and bad ways of using SOBI. NeuroImage 2005;28:507-19.

[75] Tang AC, Sutherland MT, McKinney CJ. Validation of SOBI components from high-density EEG. NeuroImage 2005;25:539-53.

[76] Tatum WO, Dworetzky BA, Schomer DL. Artifact and recording concepts in EEG. J Clin Neurophysiol 2011;28:252-63.

[77] Thatcher RW. Tutorial on adulteration of phase relations when using independent components analysis/blind identification and other regression methods to "correct for artifact", unpublished.

[78] Ting RH, Fung PCW, Chang CQ, Chan FHY. Automatic correction of artifact from single-trial eventrelated potentials by blind source separation using second order statistics only. Med Eng Phys 2006;28:780-94.

[79] Tong L, Inouye Y, Liu R. Waveform-preserving blind estimation of multiple independent sources. IEEE Trans Signal Process 1993;41:2461-70.

[80] Tran Y, Craig A, Boord P, Craig D. Using independent component analysis to remove artifact from electroencephalographic measured during stuttered speech. Med Biol Eng Comput 2004;42:627-33.

[81] Urigüen JA, Garcia B. Electroencephalogram artifact removal-validation. J Med Imaging Health Inform 2017;7:174-80.

[82] Urigüen JA and Garcia-Zapirain B. EEG artifact removal—state-of-the-art and guidelines. J Neural Eng 2015;12:031001.

[83] Vázquez RR, Velez-Perez H, Ranta R, Dorr VL, Maquin D, Maillard L. Blind source separation, wavelet denoising and discriminant analysis for EEG artefacts and noise cancelling. Biomed Signal Process Control 2012;7:389-400. 
[84] Vigário RN. Extraction of ocular artefacts from EEG using independent component analysis. Electroencephalogr Clin Neurophysiol 1997;103:395-404.

[85] Viola FC, Thorne J, Edmonds B, Schneider T, Eichele T, Debener S. Semi-automatic identification of independent components representing EEG artifact. Clin Neurophysiol 2009;120:868-77.

[86] Wallstrom GL, Kass RE, Miller A, Cohn JF, Fox NA. Automatic correction of ocular artifacts in the EEG: a comparison of regression-based and component-based methods. Int J Psychophysiol 2004;53:105-19.

[87] Weis M, Romer F, Haardt M, Jannek D, Husar P. Multi-dimensional space-time-frequency component analysis of event related EEG data using closed-form PARAFAC. In Proc. IEEE Int. Conf. Acoustics, Speech and Signal Processing ICASSP 09, p. 349-52, 2009.

[88] Welch PD. The use of fast Fourier transform for the estimation of power spectra: A method based on time-averaging over short, modified periodograms. IEEE Trans Aud Electro 1967;15:70-3.

[89] Winkler I, Brandl S, Horn F, Waldburger E, Allefeld C, Tangermann M. Robust artifactual independent component classification for BCI practitioners. J Neural Eng 2014;11:035013.

[90] Winkler I, Haufe S, Tangermann M. Automatic classification of artifactual ICA-components for artifact removal in EEG signals. Behav Brain Funct 2011;7:1-15.

[91] Zavala-Fernández H, Sander T, Burghoff M, Orglmeister R, Trahms L. Comparison of ICA algorithms for the isolation of biological artifacts in magnetoencephalography. In Int. Conf. Independent Component Analysis and Signal Separation, p. 511-8, 2006. 